\title{
Spectral modulation of exciton Fano resonance due to Zener breakdown in strongly biased superlattices
}

\author{
Ken-ichi Hino ${ }^{1,2, *}$ and Nobuyuki Toshima ${ }^{1}$ \\ ${ }_{1}^{1}$ Institute of Materials Science, Graduate School of Pure and Applied Sciences, University of Tsukuba, 1-1-1 Tennodai, Tsukuba, Ibaraki \\ 305-8573, Japan \\ ${ }^{2}$ Center for Computational Sciences, University of Tsukuba, 1-1-1 Tennodai, Tsukuba, Ibaraki 305-8577, Japan
}

(Received 20 January 2005; published 31 May 2005)

\begin{abstract}
The effects of the Wannier-Stark ladder (WSL) resonance on the optical absorption spectra of strongly biased superlattices are theoretically investigated. Exciton Fano resonance (FR) states in this system are calculated based on the multichannel scattering theory. When the bias of a static electric field $(F)$ is applied such that a WSL subband state is energetically aligned with its adjacent ones, resulting in an anticrossing with strong repulsion due to Zener resonance, we obtain the following findings. (i) The onset of exciton absorption noticeably shifts toward the lower energy side, accompanying the oscillation of the magnitude of the absorption tail with the change in $F$. However, for the anticrossing, this effect is limited to a small localized region of $F$. In fact, a slight change in $F$ away from the anticrossing leads to a peculiar suppression of the redshift of the absorption tail edge. (ii) The absorption intensities and the positions of the FR states vary in an irregular manner due to the Zener breakdown as $F$ traverses the anticrossing region. For instances in a certain WSL state, the oscillator strength is reduced by a large extent only in the anticrossing region, followed by recovery of the intensity out of this region. Moreover, the changes in Fano's $q$ values with respect to $F$ are also discussed. As described in (i) and (ii), these two effects on the exciton spectra are due to the delocalization of WSL wave functions across several periods that accompany the strong anticrossing.
\end{abstract}

DOI: 10.1103/PhysRevB.71.205326

PACS number(s): 78.66. $-\mathrm{w}, 32.80 . \mathrm{Dz}$

\section{INTRODUCTION}

Wannier ${ }^{1}$ demonstrated that, on the basis of the singleband approximation (corresponding to the Kane wave function $^{2}$ and the Houston wave function ${ }^{3}$ ), an electron in a crystal (with a spatial period of $d$ ) under a static electric field $(F)$ _termed the Wannier-Stark ladder (WSL)—has localized wave functions and discrete energy spectra with equal spacing, expressed as $\hbar \Omega_{B}=e F d$, where $\Omega_{B}$ represents the Bloch frequency, $\hbar$ represents the Planck constant divided by $2 \pi$, and $e$ represents the elementary electric charge. $\mathrm{Zak}^{4}$ and Avron et al. ${ }^{5}$ highlighted the problem underlying this assumption and proved that energy spectra are always continuous and the wave functions are neither localized nor squareintegrable due to an interband mixing effect that is ascribable to the Zener tunneling. ${ }^{6}$ The problem arising when using the WSL with the Zener effect was also discussed based on the semiclassical picture. ${ }^{7}$ Thus, the Kane approximation and the existence of equidistant energy spectra can be considered accurate only if the spectral broadening due to the Zener breakdown is much smaller than $\hbar \Omega_{B}$ for a very weak bias of $F$. However, the existence of the WSL localization and its relation with the Zener tunneling were still controversial long after. ${ }^{8-12}$

However, apart from these theoretical predictions, it was considered difficult to experimentally detect the WSL localization and the related Bloch oscillation ${ }^{13}$ due to a rather short scattering time of the carrier as compared to the temporal period of $2 \pi / \Omega_{B}$. This issue was resolved with the advent of the man-made semiconductor superlattices (SLs) by Esaki and $\mathrm{Tsu}^{14}$ due to a larger $d$ of the order of $100 \AA$. In fact, the equidistant WSL energy spectra were measured by photoluminescence and photocurrent spectroscopy, ${ }^{15,16}$ and the Bloch oscillation was measured by time-resolved fourwave mixing spectroscopy. ${ }^{17}$

The effects of the Zener breakdown under a relatively strong bias on the WSL spectra and nonlinear transport, (i.e., the Bloch oscillation) resulting in negative differential resistance (NDR), were later discussed by many authors. ${ }^{18-36}$ Hereafter, the resonant Zener tunneling between different energetically aligned subbands will be termed as Zener resonance or WSL resonance. Delocalized electron states in coupled WSL in biased SLs were first observed by photocurrent spectroscopy along with the measurement of the currentvoltage characteristics by Schneider et al. ${ }^{18}$ They observed the delocalization of electrons and anticrossing behavior ascribable to the nearest- and the second-nearest-neighbor resonant couplings induced by the Zener resonance. The third-nearest-neighbor resonant coupling was also measured by electroreflection spectroscopy. ${ }^{19}$ Resonant tunneling across a maximum of five barriers was also observed in the current-voltage characteristics. $^{20}$ These interacting WSL states were confirmed by a two-confined-subband model. ${ }^{21-24}$ Moreover, the transitions into continuum states in strongly coupled WSL were also observed by infrared spectroscopy, ${ }^{25}$ and were theoretically investigated in comparison with the Kane approximation..$^{26-28}$ In addition, the Bloch oscillation damped by the Zener breakdown that was associated with linewidth broadening due to field-induced delocalization was observed using the time-resolved measurement. ${ }^{29}$ On the other hand, the Zener resonance was studied by the analysis of the photocurrent-voltage characteristics and photoluminescence spectroscopy by addressing the connection with the electric field domain formation, ${ }^{30,31}$ and 
through dc and microwave transport experiments. ${ }^{32}$ The coexistence of WSL localization and NDR for electrons was also experimentally demonstrated. ${ }^{33}$ Furthermore, midinfrared electroluminescence from coupled WSL was generated through both intraladder and interladder transitions in the two regions of strong WSL localization and WSL resonance delocalization. ${ }^{34}$ The transition between coherent and incoherent transport in WSL was observed at different bias conditions by hot electron spectroscopy. ${ }^{35}$ Additionally, the relation between the Zener tunneling and WSL states was established by a general multiband and multichannel scattering theory in a bulk semiconductor. ${ }^{36}$

Although an exciton effect was overlooked in most of the abovementioned studies, this effect is ascertained to be significant in the linear optical transitions of WSL, ${ }^{37,38}$ specifically for the manifestation of the Fano resonance (FR) spectra $^{39}$ with characteristic asymmetric profiles. ${ }^{40-42}$ Moreover, the anomalous phenomena of WSL excitons, for example, a prolonged lifetime of intraband polarization coherence in the THz radiation and an asymmetric Autler-Townes doublet observed by spectrally resolved four-wave mixing spectroscopy, are also observed in nonlinear optical responses. ${ }^{43-47}$ Nevertheless, these studies are limited to WSL under a relatively weak bias, where the Zener breakdown is negligible and the Kane approximation can be safely applied. To the best of our knowledge, investigations on the FR spectra of WSL excitons under a strong bias are quite limited in number. ${ }^{48,49}$

The present paper demonstrates that the optical absorption spectra due to excitonic FR in WSL are strongly modulated by the alteration of the axial bias applied in the direction of crystal growth due to the Zener breakdown. When neighboring WSL subband states are energetically aligned with each other and the concomitant Zener resonance causes strong repulsion of the concerned subband energies, the following interesting effects can be observed: (i) The absorption tail edge shifts noticeably toward the lower-energy side and the absorption in the higher-energy region (above the tail) is reduced. This phenomenon can be observed in greater detail when the magnitude of the absorption tail varies with respect to $F$ in an oscillating manner. Interestingly, this effect occurs in the vicinity of the anticrossing; hence, it is suppressed away from this localized region. The preliminary discussion on the redshift of the absorption tail edge was made in Ref. 49. (ii) Since the WSL subband wave functions are drastically modified, both the intensity and the position of the FR spectral peak are also altered in an irregular manner as $F$ traverses the anticrossing region. The Zener resonance also affects the shapes of the FR spectra represented by Fano's $q$ values.

This paper is organized as follows: Sec. II presents methods of calculations. Section II A briefly discusses the numerical method for solving the multichannel scattering problem of excitons in the coupled WSL as this has already been dealt with in detail in the previous papers. ${ }^{47,50}$ Further, the method of calculating the bound and continuum subband states of the WSL is also provided. The boundary condition for the WSL problem is discussed in Sec. II B. Section II C presents, for subsequent discussion, the energy-fan diagrams and the associated wave functions of the subband states ob- tained by following the numerical methods described in Sec. II A. In Sec. II D, the FR spectra corresponding to the three existing results ${ }^{26,38,41}$ are calculated and compared with the reported spectra in order to demonstrate feasibility and validity of the present method of calculations. The results and discussion are presented in Sec. III, where the calculated FR spectra are first examined in a general overview (Sec. III A); this is followed by a more detailed discussion on both the redshift of the absorption tail edge (Sec. III B) and the spectral modulation (Sec. III C) due to the Zener resonance. Section IV is the summary. This paper also includes three appendices. In Appendix A, as a supplement to Sec. II A, a method for calculating the WSL subband wave functions is introduced based on the basis-spline (B-spline) collocation method (BSCM) ${ }^{51,52}$ and the Sturmian function expansion method. ${ }^{53,54}$ Appendix B, as a supplement to Sec. II C, examines the numerical convergence of the WSL energies and wave functions. In Appendix C, as a supplement to Sec. III B, a mathematical formulation of the free spectra is developed for a more detailed examination of the redshift of the absorption tail edge.

The sample of undoped [001] SLs employed for the calculations is $\mathrm{GaAs} / \mathrm{Ga}_{1-x} \mathrm{Al}_{x} \mathrm{As}$ of $35 / 11$ monolayers (ML) $[1 \mathrm{ML}=2.83 \AA]$ for the well and barrier thickness with $x$ $=0.25$; this is similar to the sample used in Ref. 32. The excitation densities of carriers by optical transitions are assumed to be so low that the applied electric field is homogeneous throughout the SLs, and the formation of the electric field domain ${ }^{30,31}$ is neglected. A set of quantum numbers of a WSL subband state is given by $j_{e} \equiv n_{e}\left(b_{e}\right)\left[j_{h} \equiv n_{h}\left(b_{h}\right)\right]$, where $b_{e}\left[b_{h}\right]$ and $n_{e}\left[n_{h}\right]$ are a miniband index and a WSL index of an electron [a hole], respectively. These quantum numbers, based on the Kane representation, will be adopted hereafter for the convenience of designating the quantum state, though, in actuality, meaningless for strongly coupled WSL. In order to avoid a notational confusion, the label $n_{e}\left[n_{h}\right]$ of $j_{e}\left[j_{h}\right]$ is sometimes expressed as ' $n_{e}^{\prime}\left(b_{e}\right)\left[\right.$ [ $\left.n_{h}^{\prime}\left(b_{h}\right)\right]$. Similarly, the notation, $j \equiv n\left(b_{e}, b_{h}\right)$, where $n=n_{h}-n_{e}$ is used to represent the joint WSL subband state of the electron and the hole. Hereafter, this will be termed as a channel. Atomic units are used throughout this paper unless stated otherwise. The following units are used in this paper: 1 atomic unit is equivalent to $0.52918 \AA$ for length, $27.2116 \mathrm{eV}$ for energy, and $5.1422082 \times 10^{6} \mathrm{kV} / \mathrm{cm}$ for electric field. Additionally, the empirical parameters employed here are listed in Table I for an electron mass $m_{e}$, the Luttinger parameters $\gamma_{i}(i=1-3)$, an energy band gap $E_{g}$, and a static dielectric constant $\epsilon . m_{e}, \gamma_{i}$, and $E_{g}$ of the alloy $\mathrm{Ga}_{1-x} \mathrm{Al}_{x} \mathrm{As}$ are linearly interpolated as a function of the Al concentration $x$ between the values of GaAs and AlAs. A band offset rule is adopted by which $68 \%$ of the total band gap difference between GaAs and $\mathrm{Ga}_{1-x} \mathrm{Al}_{x} \mathrm{As}$ is allocated to the conduction subband.

\section{METHODS OF CALCULATIONS}

\section{A. Subband states and FR states of the coupled WSL}

For the system under consideration, a set of coordinates $\left\{\rho, z_{e}, z_{h}\right\}$ is defined, where $\rho=(|\rho|, \phi)$ represents the in-plane 
TABLE I. Empirical parameters employed in the calculations. $E_{g}$ is expressed in the unit of $\mathrm{eV}$.

\begin{tabular}{lcc}
\hline \hline & GaAs & AlAs \\
\hline$m_{e}$ & 0.0665 & 0.15 \\
$\gamma_{1}$ & 6.790 & 3.790 \\
$\gamma_{2}$ & 1.924 & 1.230 \\
$\gamma_{3}$ & 2.681 & 1.395 \\
$E_{g}$ & 1.519 & 2.766 \\
$\epsilon$ & 12.5 & 12.5 \\
\hline \hline
\end{tabular}

relative vector between an electron $(e)$ and a hole $(h)$, with $\phi$ representing the in-plane angular coordinate, and $z_{e}$ and $z_{h}$ represent the $z$ coordinates of $e$ and $h$, respectively. Here, the center-of-mass motion of an exciton in the plane of the layer is removed. Moreover, valence-band mixing is neglected and, for simplicity, the contribution of light holes $(l h)$ is omitted, so that only the $s$-radial symmetry of the heavy hole $(h h)$ exciton is taken into account, unless stated otherwise. The effective-mass Hamiltonian $H_{e x}$ for the WSL exciton is expressed as

$$
H_{e x}\left(\rho, z_{e}, z_{h}\right)=-\frac{1}{2 m_{\|}} \nabla_{\rho}^{2}+V\left(\rho, z_{e}-z_{h}\right)+h_{w s l}\left(z_{e}, z_{h}\right),
$$

where $V$ is the Coulomb potential between them, expressed as

$$
V\left(\rho, z_{e}-z_{h}\right)=-\frac{1}{\epsilon \sqrt{\rho^{2}+\left(z_{e}-z_{h}\right)^{2}}} .
$$

In addition, $h_{w s l}$ represents the Hamiltonian of the WSL for a combined subband of $e$ and $h$, and is defined as

$$
h_{w s l}\left(z_{e}, z_{h}\right)=\sum_{k=e, h} h_{w s l}^{(k)}\left(z_{k}\right) \text {. }
$$

Here,

$$
h_{w s l}^{(k)}\left(z_{k}\right)=-\frac{\partial}{\partial z_{k}}\left(\frac{1}{2 m_{z}^{(k)}}\right) \frac{\partial}{\partial z_{k}}+u_{k}\left(z_{k}\right) \mp F z_{k},
$$

where it is understood that the arithmetic operator before the last term is "-" when $k=e$ and " + " when $k=h$, and $u_{e / h}$ is the periodic potential of the associated SLs for the motion of the particle $e / h$. Moreover, $m_{z}^{(e / h)}$ is the mass of the particle $e / h$ in the $z$ direction, and $m_{\|}$is the in-plane reduced mass of $e$ and $h$, where $1 / m_{z}^{(h)}=\gamma_{1}-2 \gamma_{2}$ and $1 / m_{\|}=\gamma_{1}+\gamma_{2}+1 / m_{e}$. It should be noted that $m_{z}^{(e / h)}$ and $m_{\|}$are functions of $z_{e}$ and $z_{h}$. In particular, the latter mass plays a key role in the massmixing effect, as will be discussed in Sec. III B.

An exciton envelope function $\Psi$ following the effectivemass model satisfies the Wannier equation

$$
\left(H_{e x}-E\right) \Psi(|\rho|, \Omega)=0,
$$

where $E$ is a given energy of the FR exciton, and $\Omega$ has been defined as a lump of the coordinates $\Omega=\left(\phi, z_{e}, z_{h}\right)$ for convenience of presentation. According to the previous formalism of Ref. 50, the $\alpha$ th solution of $\Psi_{\alpha}$ is expanded with respect to a set of adiabatic channel functions $\left\{\Phi_{\mu}\right\}$ given by the following equation:

$$
\Psi_{\alpha}(|\rho|, \Omega)=\frac{1}{\sqrt{|\rho|}} \sum_{\mu} \Phi_{\mu}(|\rho| ; \Omega) F_{\mu \alpha}^{E}(|\rho|) .
$$

Here, $\Phi_{\mu}$ is an eigenfunction of the Schrödinger equation

$$
\left(h_{w s l}+V\right) \Phi_{\mu}(|\rho| ; \Omega)=U_{\mu}(|\rho|) \Phi_{\mu}(|\rho| ; \Omega),
$$

where the in-plane radius of the exciton $|\rho|$ is fixed as an adiabatic parameter. The eigenvalue of $U_{\mu}$ represents the adiabatic potential, which is identical to the $\mu$ th WSL subband energy associated with $h_{w s l}$ at the limit $|\rho| \rightarrow \infty$ [see Eq. (14)]. An open [closed] channel $\mu$ is defined as an adiabatic channel satisfying $E>U_{\mu}(\infty)\left[E<U_{\mu}(\infty)\right]$. A radial wave function $F_{\mu \alpha}^{E}$ is provided by the use of the $R$-matrix propagation technique. ${ }^{50}$

The dipole moment $\tilde{\mu}_{\alpha}(E)$ for an interband transition to an FR state in the $\alpha$ th open channel at a given $E$ is provided by

$$
\tilde{\mu}_{\alpha}(E)=\mu_{0} \mu_{\alpha}(E) .
$$

Here, $\mu_{0}$ represents the dipole moment of an interband transition of the bulk crystal GaAs. $\mu_{\alpha}(E)$ represents the contribution of an exciton envelope function to $\tilde{\mu}_{\alpha}(E)$ :

$$
\mu_{\alpha}(E)=\sum_{\mu}^{N} w_{\mu}\left[|\rho|^{-1 / 2} F_{\mu \alpha}^{E}(|\rho|)\right]_{|\rho|=0},
$$

where

$$
w_{\mu}=\int d z\left[\Phi_{\mu}(0 ; \Omega)\right]_{z \equiv z_{e}=z_{h}},
$$

and $N$ represents the total number of channels included. The concerned photoabsorption spectra are proportional to the quantity of $I(E)$ and are expressed as

$$
I(E)=\sum_{\alpha}^{N_{0}}\left|\tilde{\mu}_{\alpha}(E)\right|^{2},
$$

where $N_{0}$ represents the total number of open channels at the photon energy of $E$.

Equation (7) is solved by expanding the adiabatic channel function $\Phi_{\mu}$ in the following equation:

$$
\Phi_{\mu}(|\rho| ; \Omega)=\sum_{j}^{N} \varphi_{j}(\Omega) c_{j \mu}(|\rho|),
$$

with respect to a set of wave functions $\left\{\varphi_{j}\right\}$ defined by

$$
\varphi_{j}(\Omega)=\frac{1}{\sqrt{2 \pi}} \exp (i m \phi) \phi_{j}\left(z_{e}, z_{h}\right) .
$$

Here, $\phi_{j}$ is a WSL subband wave function satisfying the equation

$$
\left(h_{w s l}-\varepsilon_{j}\right) \phi_{j}\left(z_{e}, z_{h}\right)=0,
$$

where the index $j$ represents the subband state of the combined WSL of $e$ and $h$, and is given by $j=n\left(b_{e}, b_{h}\right)$ based on 
the approximate Kane representation defined in Sec. I. The prefactor of $(1 / \sqrt{2 \pi}) \exp (i m \phi)$ in Eq. (13) represents a wave function of an in-plane angular motion of the $e$ and $h$ pair having an angular momentum $m$. In actuality, an excitonic photoabsorption is dominated by the $s$-radial symmetry, i.e., $m=0$; hereafter, this condition will be applied. In Eq. (12), the coefficient $\left\{c_{j \mu}\right\}$ is determined at each $|\rho| . \phi_{j}$ is expressed as a combined WSL subband wave function defined by ${ }^{37}$

$$
\phi_{j}\left(z_{e}, z_{h}\right)=\frac{1}{\sqrt{N_{\text {site }}}} \sum_{l} \exp \left(i K_{z} l d\right) \phi_{l\left(b_{e}\right)}^{(e)}\left(z_{e}\right) \phi_{l^{\prime}+n^{\prime}\left(b_{h}\right)}^{(h)}\left(z_{h}\right),
$$

where $N_{\text {site }}$ is the number of WSL sites included in the calculations, since there is an additional translational symmetry due to the center-of-mass motion of a pair of $e$ and $h$ in the $z$ direction, which conserves the associated center-of-mass momentum $K_{z}$. Hereafter, it is understood that $K_{z}$ is set to zero since only $K_{z}=0$ contributes to photoabsorption. ${ }^{37} \phi_{j_{k}}^{(k)}$ $(z)$ is a WSL wave function, where $k=e, h$, satisfying

$$
\left(h_{w s l}^{(k)}-\epsilon_{j_{k}}\right) \phi_{j_{k}}^{(k)}(z)=0,
$$

where the notation $j_{k}=n_{k}\left(b_{k}\right)$ is defined in Sec. I. The relations based on the Kane representation are clearly indicated such that

$$
\begin{gathered}
\phi_{n_{k}\left(b_{k}\right)}^{(k)}(z+l d)=\phi_{i_{n_{k}-l^{\prime}\left(b_{k}\right)}^{(k)}}(z), \\
\epsilon_{n_{k}\left(b_{k}\right)}=\epsilon_{n_{n_{k}-l^{\prime}\left(b_{k}\right)}} \mp l \Omega_{B}, \\
\varepsilon_{n\left(b_{e}, b_{h}\right)}=\epsilon_{n_{e}\left(b_{e}\right)}+\epsilon_{n_{h}\left(b_{h}\right)}=\varepsilon_{0\left(b_{e}, b_{h}\right)}+n \Omega_{B},
\end{gathered}
$$

where $n=n_{h}-n_{e}$. Note that the energy of $\epsilon_{n_{k}\left(b_{k}\right)}$ is always continuous $^{4}$ and that the discrete label $n_{k}$ has been adopted for convenience. In Sec. II B, this issue will be commented on in conjunction with the boundary conditions imposed on the solutions of Eq. (16).

The numerical method of solving Eq. (16) is summarized here, while its details are presented in Appendix A. We employed the following stepwise procedure to obtain a set of wave functions $\left\{\phi_{j_{k}}^{(k)}\right\}$. (i) Initially, a Sturmian basis set corresponding to a single quantum well system is calculated in advance, where the set is expanded in terms of the B-splines, ${ }^{51}$ following the BSCM. ${ }^{52}$ This Sturmian method provides both discrete and pseudocontinuum spectra on an equal footing, without the disadvantage of possessing the real continuum spectra. ${ }^{53,54}$ (ii) A Wannier function associated with the present SLs is expanded in terms of these Sturmian functions. By means of a standard algebraic equation, the SL spectra for both bound state components (below the barrier of the confining potential) and continuum components (above the barrier of the confining potential) are provided by straightforward calculations. (iii) The WSL wave functions are obtained by using these SL wave functions as an expansion basis set. Here, the notation WF- $l$ is used for approximate WSL wave functions obtained by expansion in terms of the SL wave functions ranging from the lowest miniband to the $l$ th miniband. First, WF-1 are calculated by including only the SL states pertaining to the lowest miniband and labeled as $n_{k}\left(b_{k}\right), k=e, h$ based on the Kane representation. The SL states pertaining to the first and the second lowest minibands are subsequently used for more accurate calculations of WSL wave functions of WF-2. Strictly speaking, the resulting WF-2 can no longer be classified by the Kane quantum number. However, the most suitable Kane quantum number, though approximate, can always be assigned to any state of WF-2 by comparing the eigenvalues relevant to WF-2 with those relevant to WF-1. The same procedure is repeated to obtain WF- $l$ by assigning them the Kane quantum numbers in reference to those assigned to WF- $(l-1)$. Finally, we obtain WF- $N_{b}$ that are adopted for the basis set $\left\{\phi_{j_{k}}^{(k)}\right\}$, where $N_{b}$ represents the maximum number of SL minibands included in the calculations.

However, not only does the procedure described above appear complicated, but its advantages are also rather unclear. Actually, there are other straightforward methods to solve Eq. (16). For instance, it can be solved by collocating a set of knots of B-splines over a tilted WSL potential using the generalized eigenvalue problem. It can also be solved using the Runge-Kutta method by propagating the WSL wave function from a high-potential region toward a lowpotential asymptotic region with an appropriate matching procedure in terms of a set of Airy functions. The solutions provided by these two methods are equivalent to those of the procedure, described in the steps from (i) to (iii). However, the former methods do not allow the practical classification of every wave function on the basis of the approximate Kane quantum numbers. Hence, it becomes difficult to obtain the combined WSL wave function of the pair of $e$ and $h$ in the form of Eq. (15), where the wave function $\phi_{j_{k}}^{(k)}$ needs to be described by both $n_{k}$ and $b_{k}$. This is the primary reason that the procedure described in the steps from (i) to (iii) is adopted in this study.

We now discuss some advantages of the BSCM used in step (i) over the usually adopted method, using the KronigPenny model that is limited to a rectangular periodic potential. ${ }^{38}$ The BSCM can be adopted for a wide range of potentials, regardless of whether the potential is smooth or discontinuous. In fact, for the present rectangular potential, the BSCM provides a large number of highly accurate eigenvalues in one step, by introducing knot multiplicity ${ }^{51}$ at the position corresponding to the boundary between the well and the barrier where the potential is discontinuous. With minor changes, the same computer program can be easily applied to systems such as a sinusoidal potential that models the WSL of optical lattices of ultracold atoms realized by laser-cooling technology. ${ }^{55-59}$

\section{B. Boundary conditions for the WSL problem}

Prior to the discussion on the WSL subband energies $\left\{\epsilon_{j_{k}}\right\}$ and the associated wave functions $\left\{\phi_{j_{k}}^{(k)}\right\}$, herein termed as set A, obtained by following the steps (i)-(iii) in Sec. II A, we take into consideration the boundary conditions to be imposed on the solutions of Eq. (16). The WSL subband energies thus obtained are always discrete because a limited 
number of $N_{b}$ and a finite number of $N_{\text {site }}$ are employed along with the vanishing boundary condition that is expressed as $\phi_{j_{k}}^{(k)}\left(z_{-\infty}\right)=\phi_{j_{k}}^{(k)}\left(z_{\infty}\right)=0$. Here, $z_{-\infty}$ and $z_{\infty}$ are the left and right end points, respectively, and are expressed as $z_{-\infty}=$ $-\left(N_{\text {site }} / 2\right) d$ and $z_{\infty}=\left(N_{\text {site }} / 2-1\right) d$. Instead of this vanishing boundary condition, an arbitrary boundary condition such as the fixed boundary condition of $\left[d \phi_{j_{e}}^{(e)}(z) / d z\right]_{z=z_{\infty}}=0$ can also be imposed on the wave function at the right and left end points for $k=e$ and $k=h$, respectively. However, the imposition of the different boundary condition leads to providing another set of the WSL energy spectra that are different from $\left\{\epsilon_{j_{k}}\right\}$. In this context, it could be stated that the discussion on this particular set of the energy spectra makes little sense at a glance. However, in reality, set A allows for a deep insight of physics pertaining to the behavior of couplings between $l o-$ calized Kane subband states satisfying the vanishing boundary conditions, eventually leading to field-induced delocalization.

In view of the continuum feature of the WSL spectra, the open boundary condition is more preferable, and the associated solutions, for example, set B: $\left\{\phi_{\epsilon}^{(k)}\right\}$, are practically calculated using the Runge-Kutta method mentioned in Sec. II A, where $\epsilon$ is a given energy of the continuum state. For a very large $N_{\text {site }}$, the following statement holds; or else, the open boundary condition should not be considered. Since both of sets A and B satisfy the same Schrödinger equation of Eq. (16), the Hilbert space spanned by set B is of the same size as that spanned by set A in spite of the different boundary conditions being imposed, as long as $N_{b}$ is sufficiently large. Specifically, the following closure relation exists:

$$
\sum_{j_{k}} \phi_{j_{k}}^{(k)}(z)\left[\phi_{j_{k}}^{(k)}\left(z^{\prime}\right)\right]^{*}=\int d \epsilon D(\epsilon) \phi_{\epsilon}^{(k)}(z)\left[\phi_{\epsilon}^{(k)}\left(z^{\prime}\right)\right]^{*}=\delta\left(z-z^{\prime}\right),
$$

where $D(\epsilon)$ represents the density of state determined by the normalization condition of $\phi_{\epsilon}^{(k)}$. Therefore, it is evident that both of sets A and B are mathematically equivalent. On the basis of this, it can be stated that the former set of discrete wave functions is compatible with the latter set of continuum wave functions.

Nevertheless, from a practical point of view, the discrete set $\mathrm{A}$ is even more feasible and efficient than the continuum set $\mathrm{B}$, since all the wave functions of the former set can be simultaneously provided in one calculation step by resorting to the BSCM, and possible physical meanings can be implied from every WSL subband state based on the approximate Kane representation. On the other hand, with reference to the latter set, the wave functions are provided individually by using the Runge-Kutta routine; in practical calculations, it is indispensable to discretize the entire continuum energy spectra, for instance, in terms of an energy sampling based on the Gaussian quadrature. In general, the number of the discretized basis functions of set B required to attain well convergent results of the exciton FR spectra is greater than the number of the discrete basis functions of set $\mathrm{A}$. This is because the discretized basis functions of set B are arranged in terms of the energy sampling method irrespective of the un-

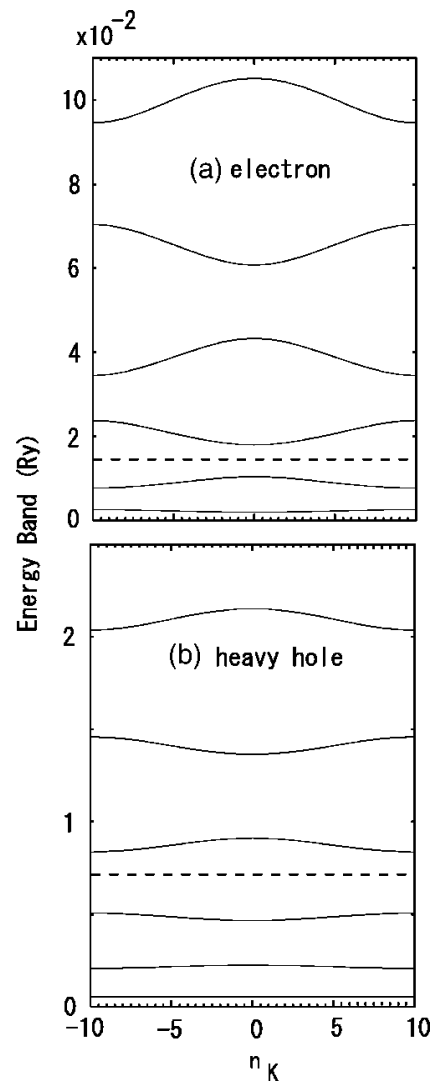

FIG. 1. Energy band structures of the present SLs for (a) the electron and (b) the heavy hole. The horizontal dashed line indicates the height of the confining potential barrier. The abscissae of $n_{K}$ are given by $n_{K}=N_{\text {site }} K d / 2 \pi$, where $K$ represents the Bloch momentum of the SLs and $N_{\text {site }}=20$.

derlying physics; hence, as a result, certain parts of the WSL subband states included in it do not contribute much to the exciton FR spectra. Therefore, it can be concluded that the basis set A adopted in this study is considered optimal.

\section{Energies and wave functions of the WSL subband states}

Figure 1 shows six energy bands $\left(b_{e / h}=1-6\right)$ of the SLs for $e$ and $h$ obtained by following step (ii). With the exception of the first lowest band, the second and third lowest bands are located below the confining potentials of $e$ and $h$, respectively. Miniband widths of $h$ are much smaller than those of $e$ due to a heavier effective mass of $h$, as is expected.

The energy-fan diagram of the coupled WSL for $e$, calculated with $N_{\text {site }}=20$ and $N_{b}=6$, is shown in Fig. 2(a) ${ }^{60}$ Prior to examining the results of this figure, a brief remark is made on the convergence of the present calculations based on Eq. (A8). Here, the convergence of numerical solutions with respect to $N_{b}$ has been observed by varying $N_{b}$. The obtained results are given in Appendix B. Furthermore, the convergence is also confirmed by comparing the results with $N_{b}$ $=6$ either with the results obtained from the direct BSCM calculations or with those obtained from the Runge-Kutta calculations mentioned in Sec. II A. As is shown in Appen- 

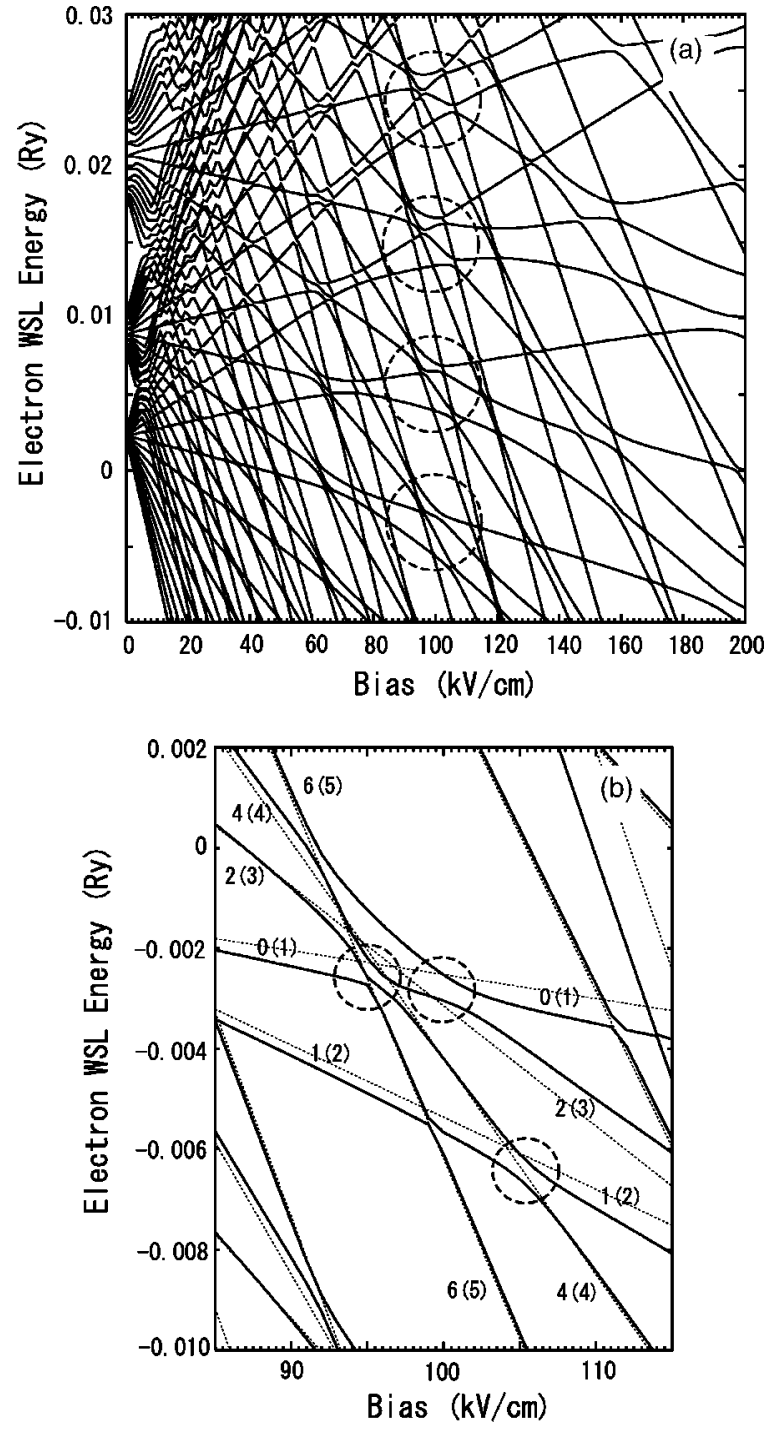

FIG. 2. (a) The energy-fan diagram $\epsilon^{(e)}$ of the coupled WSL for $e$ as a function of the bias of $F$. The lowest six minibands of the SLs $\left(N_{b}=6\right)$ are included as a basis set for the calculations, with $N_{\text {site }}$ $=20$. Dashed circles indicate a series of anticrossings in the vicinity of $F=100 \mathrm{kV} / \mathrm{cm}$. (b) Expanded view around the region indicated by the dashed circles at the bottom of panel (a). Moreover, energies calculated under the Kane approximation are also shown by dotted lines. The approximate Kane quantum numbers $n_{e}\left(b_{e}\right)$ participating in this anticrossing region are indicated. The dominant anticrossings in this region are also represented by a dashed circle each.

dix B, the energies and wave functions of the coupled WSL state of $0(1)$ to $0(4)$ indicate excellent convergence that is warranted by the Rayleigh-Ritz variational principle. ${ }^{61}$ On the other hand, while minor numerical errors are incurred in $0(5)$ and $0(6)$, these may be due to the limited number of $N_{b}$ that is practically included. It was pointed out in Ref. 26 that the convergence of the coupled WSL wave functions with respect to $N_{b}$ seems doubtful, and hence, all the orders of contributions from the interminiband interactions should be incorporated, since Eq. (A8) results in

$$
\lim _{\Delta \rightarrow 0} \sum_{B_{k}} \sum_{K} \mathcal{Z}_{K B_{k} \epsilon+\Delta}^{*} \mathcal{Z}_{K B_{k} \epsilon}=\lim _{\Delta \rightarrow 0} \delta(\Delta)=\infty,
$$

where the energy labeled as $\epsilon_{j_{k}}$ has been replaced by the corresponding continuum energy of $\epsilon$. However, despite the comment of Ref. 26, this infinity does not matter in reality because it arises simply from an energy-normalization condition for the WSL wave functions. As mentioned in Sec. II B, the set of wave functions $\phi_{j k}^{(k)}$ satisfying the vanishing boundary condition is equivalent to that of wave functions $\phi^{(k)} \epsilon$ satisfying the open boundary condition relevant to the energy-normalization condition. Therefore, in the present case, Eq. (21) is simply replaced by

$$
\sum_{B_{k}} \sum_{K}\left|\mathcal{Z}_{K B_{k} j_{k}}\right|^{2}=1,
$$

which is, needless to say, satisfied irrespective of $N_{b}$ in the formulation developed in this study. Hence, Eq. (21) does not make sense for verifying the convergence of the coupled WSL wave functions with respect to the $N_{b}$.

We now examine Fig. 2(a). Anticrossings can be observed in the vicinity of $F=70,100$, and $180-200 \mathrm{kV} / \mathrm{cm}$, where the WSL subband states are energetically aligned with each other, resulting in the Zener resonance. The series of the anticrossings at $F \approx 70 \mathrm{kV} / \mathrm{cm}$ arises primarily from the interactions between the $l(1)$ and ' $l+1^{\prime}(2)$ states. Hence, this anticrossing is due to the lowest two bound state components of the original SLs [see Fig. 1(a)]. ${ }^{32}$

On the other hand, the series of the anticrossings at $F$ $\approx 100 \mathrm{kV} / \mathrm{cm}$ (four of which are indicated by dashed circles) is primarily governed by interactions among the $l(1)$, ' $l$ $+1^{\prime}(2), ' l+2^{\prime}(3)$, and ' $l+4^{\prime}(4)$ states, where the last two states are associated with the continuum components [see Fig. 1(a)]. In Fig. 2(b), the anticrossing region-denoted by the dashed circle at the bottom of Fig. 2(a) - is expanded, and the labels of the Kane quantum numbers of the $0(1)$, 1(2), 2(3), 4(4), and 6(5) states are specified, where the WSL energies calculated under the Kane approximation are shown by dotted lines for the sake of comparison. In this region, there are three dominant anticrossings located in the vicinity of $F=95,100$, and $105 \mathrm{kV} / \mathrm{cm}$, all of which are specified by dashed circles: the first is located among the 0(1), 2(3), 4(4), and 6(5) states, the second lies between the $0(1)$ and 2(3) states, and the third lies between the 1(2) and 4(4) states. The manner of the interactions for the strongest anticrossing at $F=100 \mathrm{kV} / \mathrm{cm}$ is observed by examining each WSL wave function for $0\left(b_{e}\right)$, depicted in Fig. 3. Here the solid curve indicates the wave function of the coupled WSL and the dotted curve indicates the noninteracting Kane wave function for the purpose of comparison. The difference between these two wave functions represents the degree of magnitude of the Zener tunneling. It should be noted that a wave function of the state $n_{e}\left(b_{e}\right)$ with $n_{e} \neq 0$ is obtained from that of the state $0\left(b_{e}\right)$ in Fig. 3 by using Eq. (17). The strength of the anticrossing is determined by the overlapping of the associated wave functions; in particular, the strength is dominated by the overlapping between the $0(1)$ and 2(3) states. The dominant part of this overlap corresponds to that between the arrowed portions of the wave functions of $0(1)$ and $0(3)$ as 


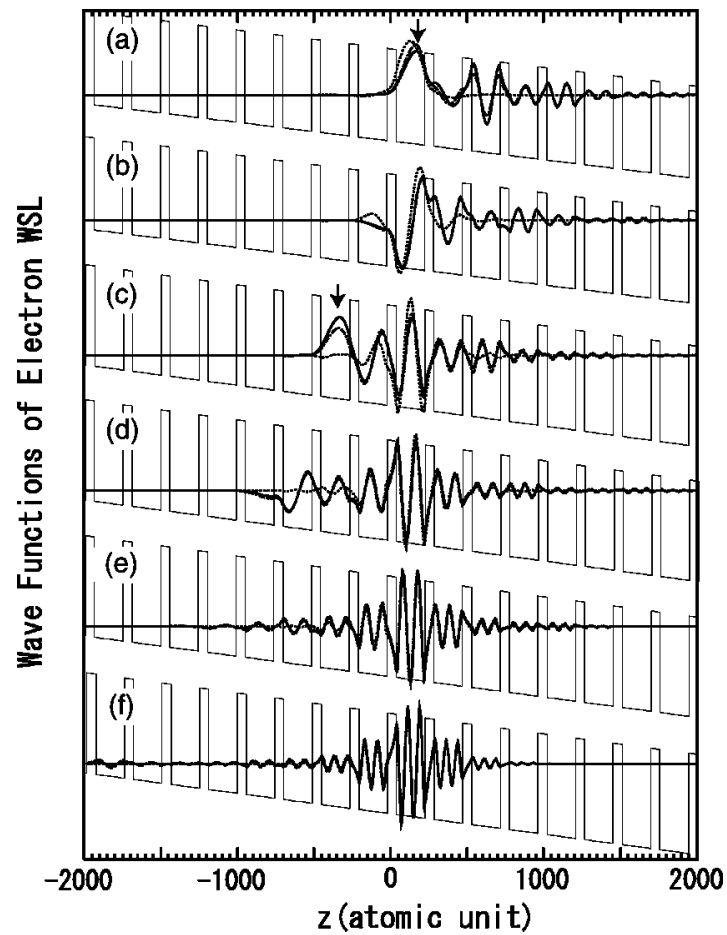

FIG. 3. The wave functions $\phi_{0\left(b_{e}\right)}^{(e)}$ of the coupled WSL for $e$ at $F=100 \mathrm{kV} / \mathrm{cm}$. These are obtained by using $N_{b}=6$ and $N_{\text {site }}=20$, and are indicated by solid lines. For the purpose of comparison, the corresponding Kane wave functions are also indicated by dotted lines. The subband indices of the wave functions are based on the approximate Kane quantum number, being labeled as $0\left(b_{e}\right)$, where $b_{e}$ of each panel is equal to (a) 1 , (b) 2 , (c) 3, (d) 4, (e) 5, and (f) 6 . Refer to the text for portions indicated by arrows in the panels (a) and (c). Additionally, the spectra, which are represented by chain lines and obtained from the model calculations, are included. Refer to the text for further details. The geometry of the present WSL is specified in each panel.

shown in Fig. 3. Therefore, it is seen that the interaction between these two states of $0(1)$ and 2(3) states causes anticrossing with conspicuous repulsion, though the other states show a relatively small amount of these effects. By further increasing $F$ to more than $150 \mathrm{kV} / \mathrm{cm}$, the anticrossings with strong repulsion extend over a wider region of the WSL energy. This is especially noticeable in the vicinity of $F$ $=190 \mathrm{kV} / \mathrm{cm}$.

The energy-fan diagram of the coupled WSL for $h$ is depicted in Fig. 4, with $N_{\text {site }}=20$ and $N_{b}=6 .{ }^{60}$ Excluding the smaller energy splittings accompanied by anticrossings, the variance of the energy with respect to $F$ is similar to that seen in Fig. 2 (for $e$ ). The series of anticrossings at $F$ $\approx 100 \mathrm{kV} / \mathrm{cm}$ arising from an interaction between the $l(1)$ and ' $l-1$ ' (4) states are indicated by dashed circles. It should be noted that the ' $l-1$ ' (4) state is pertinent to the continuum subband state of the original SLs, as is seen in Fig. 1(b). Figure 5 indicates the associated wave functions at $F$ $=100 \mathrm{kV} / \mathrm{cm}$, where the solid and dotted lines indicate the same functions as those shown in Fig. 3. A strong mixing between the 0(1) and -1(4) states is observed in view of the arrowed portions pertaining to the (a) and (d) panels in Fig.

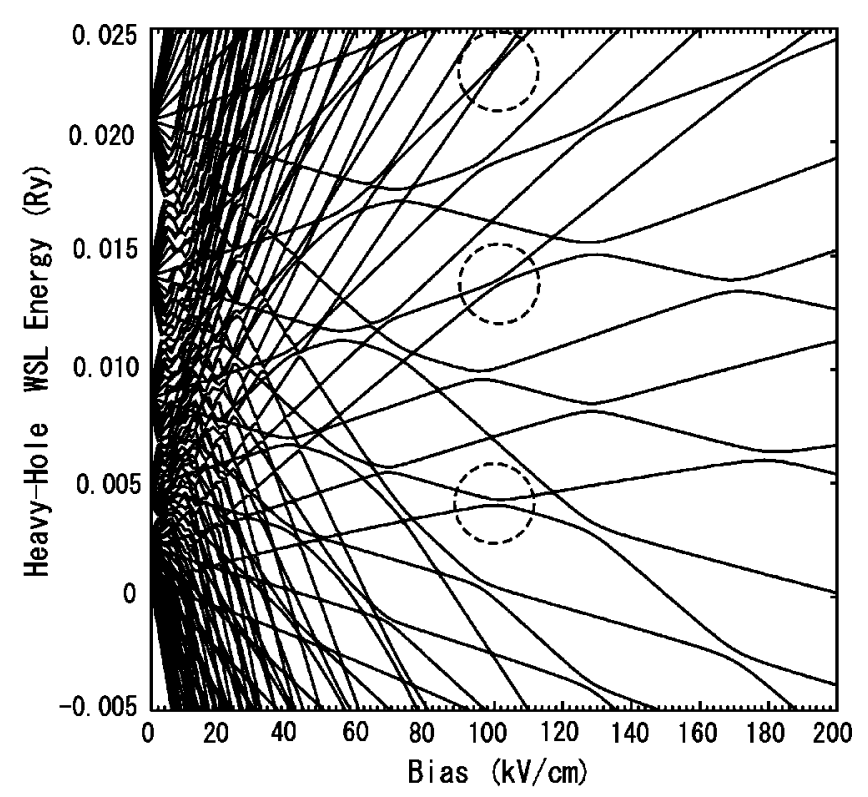

FIG. 4. The same as Fig. 2(a) but for $h$.

5. This is in contrast to our common understanding that a hole wave function is almost localized to a single well region, and compared with that of $e$, the mixing is negligible even for a relatively large $F$ due to its heavier effective mass.

\section{Demonstration of the present method}

In order to demonstrate the feasibility and validity of the present method described in Sec. II A, first, the photoabsorp-

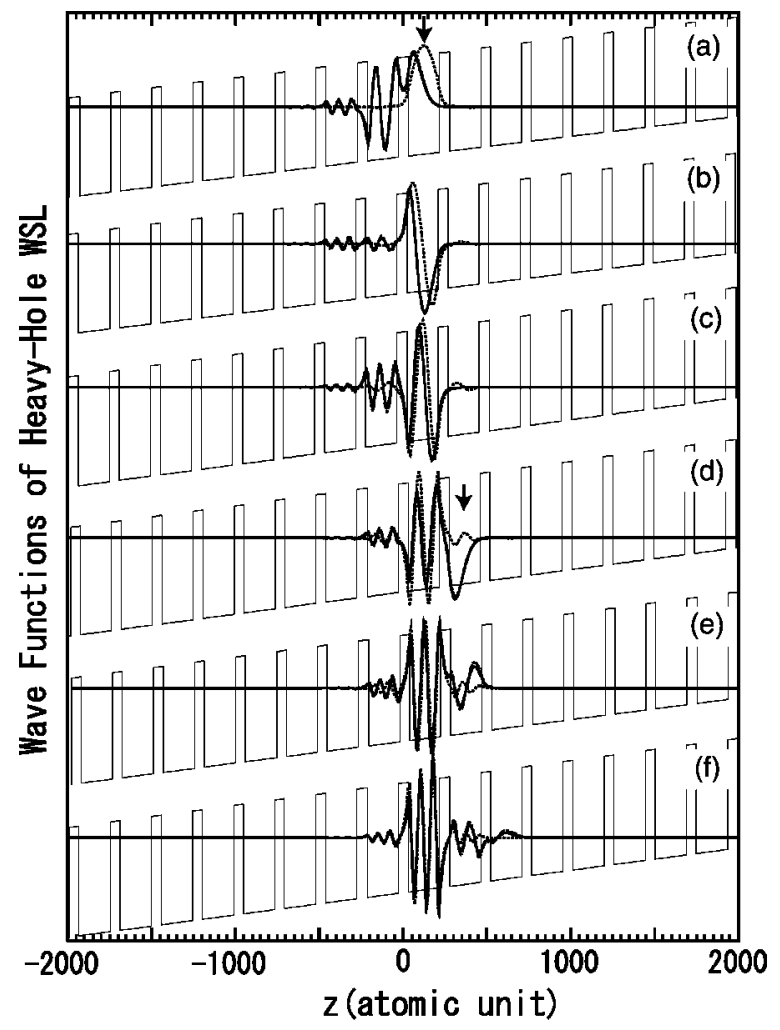

FIG. 5. The same as Fig. 3 but for $h$. 


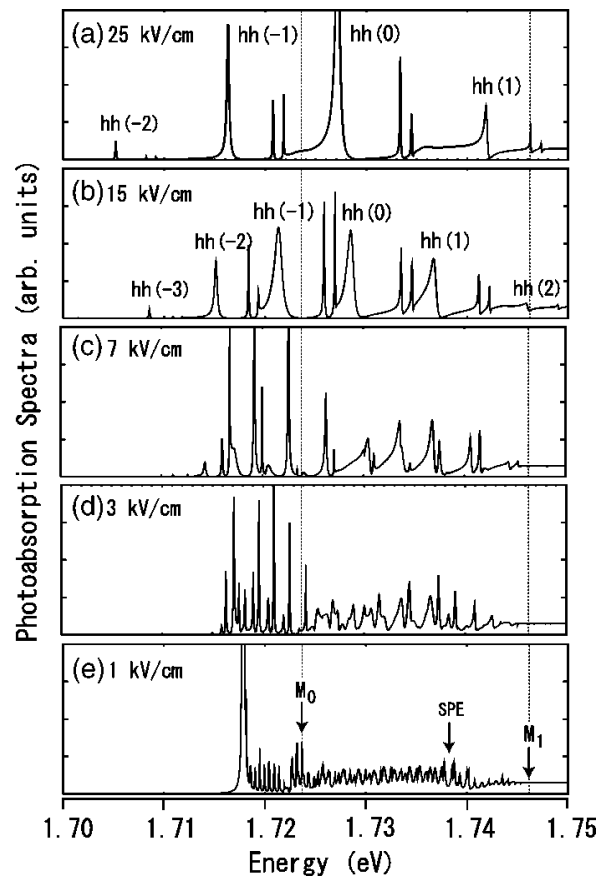

FIG. 6. Excitonic dimensionality transitions of FR spectra between the SLs and the WSL for the biased SLs of 12/6 ML GaAs/AlAs with respect to $F$ in such a small bias region that the Kane approximation is still correct, where $F=$ (a) $25 \mathrm{kV} / \mathrm{cm}$, (b) $15 \mathrm{kV} / \mathrm{cm}$, (c) $7 \mathrm{kV} / \mathrm{cm}$, (d) $3 \mathrm{kV} / \mathrm{cm}$, and (e) $1 \mathrm{kV} / \mathrm{cm}$. The spectra calculated with $N_{\text {site }}=90$ are convoluted by the Lorentzian function with $\Gamma=0.1 \mathrm{meV}$. This system of the SLs is the same as that observed in Fig. 2(a) of Ref. 38. Refer to the text for the label of $h h(n)$ in panels (a) and (b). The locations of the $M_{0}$ and $M_{1}$ Van Hove critical points of the original SLs are indicated by dotted vertical lines, and are further indicated by arrows in panel (e), where the location of the saddle point exciton (SPE) is also specified.

tion spectra of the same SL system as that used for Fig. 2(a) of Ref. 38, i.e., the 12/6-ML GaAs/AlAs SLs, are calculated for a wide range of $F$, as is shown in Fig. 6. Here, a $1 s$ state of $h h$-exciton FR pertaining to the $n(1,1)$ channel is labeled as $h h(n)$, as shown in Figs. 6(a) and 6(b), and the locations of $M_{0}$ and $M_{1}$ Van Hove critical points of the original SLs, for example, $\varepsilon_{M_{0}}$ and $\varepsilon_{M_{1}}$, respectively, are indicated by dotted lines, and arrowed especially in Fig. 6(e). Note that $\Omega_{B}$ $<\Delta_{1}$ in the concerned range of $F$ so that the Zener tunneling is negligible, where $\Delta_{1}$ is the width of the lowest joint miniband of the SLs; i.e., $\Delta_{1}=\left|\varepsilon_{M_{1}}-\varepsilon_{M_{0}}\right|=22.4 \mathrm{meV}$. The resulting natural spectra are convoluted by the Lorentzian function with the full width at half-maximum (FWHM) $\Gamma$ $=0.1 \mathrm{meV}$, which is much smaller than a typical linewidth of FR of the order of $\sim 1 \mathrm{meV} .{ }^{50}$ The alteration of these almost natural FR spectra with respect to $F$ is traced in order to observe the dimensionality transition between the threedimensional limit $(F=0)$ and the quasi-two-dimensional limit (a large $F$ ). This purpose also motivated the calculations provided in Refs. 38 and 40. It is remarked that in the theoretical method of Ref. 38 the multichannel continua characteristic of FR were approximated by a discrete basis set and the proper scattering boundary conditions were dis- regarded from the beginning. Thus, obtaining a natural FR spectra seems impossible based on this method, since such an incomplete treatment of FR always requires an additional empirical broadening. In fact, in Ref. 38, the Gaussian broadening with $\Gamma=3 \mathrm{meV}$ was used, leading to the masking of the detailed change in the FR spectra. The drawbacks that are ascribable to such conventional methods for FR have been discussed in detail in Refs. 40 and 50. Incidentally, for a positive (negative) $q$, a spectrum has a dip (a peak), followed by a peak (a dip); the spectrum with $|q| \gg 1$ is Lorentzian, and that obtained with $q=0$ has a transparent window (a dip) without any peak. ${ }^{39,62}$

Although the results obtained in Fig. 6 are similar in part to those of Ref. 40, we will now provide a detailed explanation on the spectral modulation shown in this figure. In the case of relatively large biases of $F=25 \mathrm{kV} / \mathrm{cm} \quad\left(\Omega_{B}\right.$ $=12.75 \mathrm{meV})$ and $F=15 \mathrm{kV} / \mathrm{cm}\left(\Omega_{B}=7.65 \mathrm{meV}\right)$ in Figs. 6(a) and 6(b), respectively, individual Rydberg series of FR exciton states are clearly distinguishable, and each spectrum is seen to possess a peak followed by a dip with a negative Fano $q$ value. Moreover, the characteristic exciton asymmetry between the $h h(-1)$ and $h h(1)$ spectra is manifested. ${ }^{37} \mathrm{As}$ is shown in Fig. 6(c), with $F$ decreasing to $7 \mathrm{kV} / \mathrm{cm}\left(\Omega_{B}\right.$ $=3.57 \mathrm{meV}$ ), the positions of the main peaks are so close that each Rydberg series is partially overlapped with its adjacent one belonging to a different channel; each series, however, is still barely discernible. It is noteworthy that the energy of $\varepsilon_{M_{0}}$ sharply delimits the boundary of two different patterns of spectral modulation. In the photon energy region $E$ from $\varepsilon_{M_{0}}$ to $\varepsilon_{M_{1}}$, apart from the $q$ values of the spectra remaining negative, the linewidths are obviously broadened accompanying the reduction of the intensities. This is presumably due to constructive interference with adjacent FR spectra. Here, most of all the spectra thus featured are situated within the energy region of $\varepsilon_{M_{0}}<E<\varepsilon_{M_{1}}$. In contrast, in the region $E<\varepsilon_{M_{0}}$, the linewidths are prominently narrowed, accompanying the enhancement of the intensities; this is presumably due to destructive interference with adjacent FR spectra. In this case, by constructive (destructive) interference, it can be considered that the overlap resonance between the FR spectra pertaining to different channels ${ }^{63}$ has a tendency to enhance (diminish) the overall background continuum.

As shown in Fig. $6(\mathrm{~d})$, at $F=3 \mathrm{kV} / \mathrm{cm}\left(\Omega_{B}=1.53 \mathrm{meV}\right)$, the abovementioned tendency observed in Fig. 6(c) is even more pronounced. In particular, in the region $E>\varepsilon_{M_{0}}$, the overlap resonance causes rather complicated spectral profiles; thus it is impossible to assign each peak to an appropriate exciton state, and the background continua ascribable to the individual FR spectra seem to be amalgamated into a single broad continuum by the constructive interference. Furthermore, an envelope formed by a series of FR peaks in $E$ $>\varepsilon_{M_{0}}$ appears to oscillate; this is reminiscent of the FranzKeldysh oscillation discussed in Ref. 38. As seen in Fig. $6(\mathrm{e})$, at $F=1 \mathrm{kV} / \mathrm{cm}\left(\Omega_{B}=0.51 \mathrm{meV}\right)$, the overall spectral shape becomes almost identical to that of the original SLs, consisting of a Rydberg series of exciton bound states with strong intensities below $\varepsilon_{M_{0}}$, followed by a broad continuum characterized by both the $M_{0}$ and $M_{1}$ critical points. These 
distinct exciton peaks are a result of the coalescence of a large number of fine spectra with larger intensities and narrower widths than those observed at $F=3 \mathrm{kV} / \mathrm{cm}$, which are caused by the abovementioned destructive interference. On the other hand, the broad continuum arises from the coalescence of a large number of fine spectra with smaller intensities and broader widths than those observed at $F=3 \mathrm{kV} / \mathrm{cm}$, which are caused by the abovementioned constructive interference. Additionally, a faint hump is discerned just below the slight dip at approximately $1.74 \mathrm{eV}$, which is relevant to the $M_{1}$ critical point modified by an exciton effect to a certain extent. This hump is considered as a sign of the formation of a saddle point exciton, which is a typical characteristic of SL exciton states. The excitonic dimensionality transition between the SLs and the WSL is likely to be understood as a spectral modulation of FR, as is described above.

As the second demonstration, a comparison is made with the experimental spectra of the $67 / 17-\AA$ GaAs $/ \mathrm{Ga}_{0.7} \mathrm{Al}_{0.3}$ As SLs with $F=13.3 \mathrm{kV} / \mathrm{cm}$ given in Ref. 41. The calculated spectra convoluted by the Lorentzian function with $\Gamma=0.25 \mathrm{meV}$ is shown in Fig. 7(a), where the contributions of the $t h$ excitons are also included. In this case, each peak of an $\mathcal{N} s$-state pertaining to the $h h(n)[\operatorname{lh}(n)]$ channel is labeled by $h h(n)[\mathcal{N}]\{\operatorname{lh}(n)[\mathcal{N}]\}$. This label of $\mathcal{N}$ is omitted for the sake of simplicity, in the case that there is no confusion. The same spectra as those of Fig. 7(a) but with $\Gamma=1.5 \mathrm{meV}$ are shown in Fig. 7(b), and the reported experimental spectra-labeled E-to be compared with these are cited in Fig. 7(c). On the basis of the method used in Ref. 40, the theoretical spectra are also included in Fig. 7(c) and labeled as $\mathrm{T}$. In order to aid the presentation, eight dotted vertical lines are depicted in Figs. 7(b) and 7(c) and these lines indicate the positions of the absorption peaks for the $h h(-3), \quad h h(-2)[1], \quad h h(-1)[1]+\ln (-3), \quad h h(-1)[2]$, $h h(-1)[3], h h(0)[1], h h(0)[2]$, and $h h(1)[1]$ transitions by following the peak positions labeled in Fig. 7(a). The value $(1.5 \mathrm{meV})$ of the FWHM has been employed in Fig. 7(b) for a good reproduction of the experimental width of the main peak of $\{h h(-1)[1]+l h(-3)\}$ in the E spectra. Further, the overall spectral intensity of Fig. 7(b) is also normalized to the height of this peak. By comparing the calculated spectra shown in Fig. 7(b) with the E spectra, a slight redshift by about $2 \mathrm{meV}$ is observed at every peak position of the former spectra; this discrepancy would arise from the empirical parameters adopted here in Table I. Furthermore, according to the calculated results in Fig. 7(b), the peak observed at approximately $1.579 \mathrm{eV}$ in the $\mathrm{E}$ spectra is assigned to a lump of the subbands of $h h(-1)[\mathcal{N} \geqslant 2]$ in view of the abovementioned redshift, whereas it was claimed that it arose from the $l h(-2)$ transition in Ref. 41 . The peak of the $\operatorname{lh}(-2)$ transition in Fig. 7(b) is located in the higher-energy side by about $5 \mathrm{meV}$, as compared with the peak located at approximately $1.579 \mathrm{eV}$ in the E spectra. Since this is a blueshift in the opposite direction to the abovementioned redshift, the assignment of the peak concerned in the E spectra to the $l h(-2)$ transition appears questionable. Apart from these discrepancies, it is observed that the peak positions, the spectral profiles, and the height of the background continuum

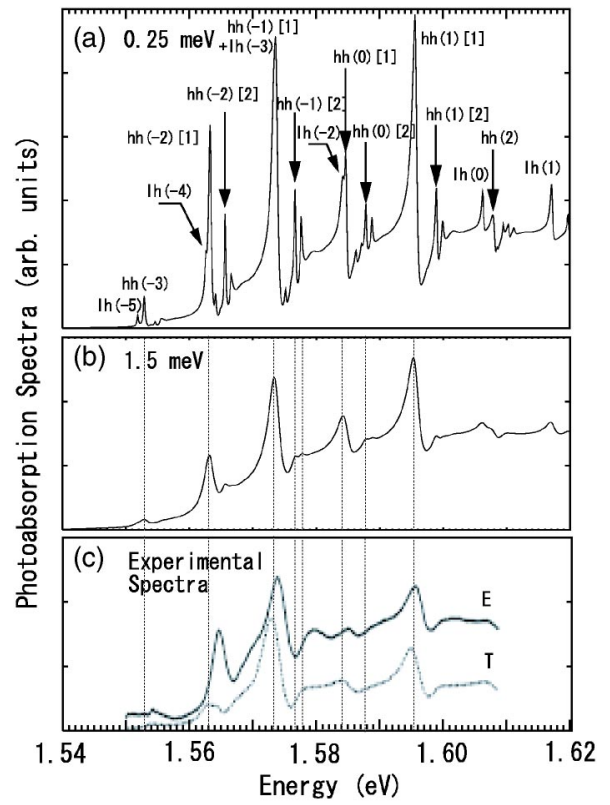

FIG. 7. The FR spectra of the $67 / 17-\AA \mathrm{GaAs} / \mathrm{Ga}_{0.7} \mathrm{Al}_{0.3} \mathrm{As}$ with $F=13.3 \mathrm{kV} / \mathrm{cm}$ as a function of photon energy $E$. This system is the same as that of Ref. 41. (a) The calculated spectra with $N_{\text {site }}$ $=20$ and the Lorentzian broadening of $\Gamma=0.25 \mathrm{meV}$. Refer to the text for the meanings of the labels. (b) The same as panel (a) but with $\Gamma=1.5 \mathrm{meV}$. (c) The experimental spectra reported in Ref. 41, labeled by E. For the purpose of comparison, the calculated spectra included in this reference are also shown with label T. In order to aid the presentation, eight dotted vertical lines are depicted in panels (b) and (c) and these indicate the positions of the absorption peaks for the $h h(-3), h h(-2)[1], h h(-1)[1]+l h(-3), h h(-1)[2]$, $h h(-1)[3], h h(0)[1], h h(0)[2]$, and $h h(1)[1]$ transitions by following the peak positions labeled in panel (a). The spectra given in panel (b) are convoluted and normalized in order to reproduce the spectral width and height of the main peak of $[h h(-1)[1]+\operatorname{lh}(-3)]$ in panel (c), respectively.

are well reproduced in the calculated spectra. When the T spectra are compared with the present spectra, the height of the background continuum is too small; the reasons for this, however, are unknown.

The last demonstration is devoted to a comparison of the present calculated spectra, denoted by solid lines in Fig. 8(a), with the existing theoretical spectra, denoted by the darkgray area in Fig. 8(b). The latter is cited from Ref. 26, where the concerned system is the $111 / 17-\AA \mathrm{GaAs} / \mathrm{Ga}_{0.7} \mathrm{Al}_{0.3} \mathrm{As}$ SLs with $F=8,40$, and $80 \mathrm{kV} / \mathrm{cm}$. $\Gamma=2 \mathrm{meV}$ is adopted in both the calculated spectra. Further, the spectra convoluted with $\Gamma=0.3 \mathrm{meV}$ are also represented by dotted lines in Fig. 8(a) solely for the purpose of assigning the discernible peaks to proper exciton $1 s$-states labeled by $n\left(b_{e}, b_{h}\right)$. As shown in Fig. 8(b), other traces of spectra with black and light-gray areas for the optical density of states and the spectra of the bulk GaAs, respectively, are not necessary for the present discussion. Regarding the method of calculating the FR spectra of Fig. 8(b), it is stated that the theoretical drawbacks similar to those commented on in the discussion of Fig. 6 would be more or less incurred. At $F=8$ and $40 \mathrm{kV} / \mathrm{cm}$, both spectra given in Figs. 8(a) and 8(b) are in good agreement 

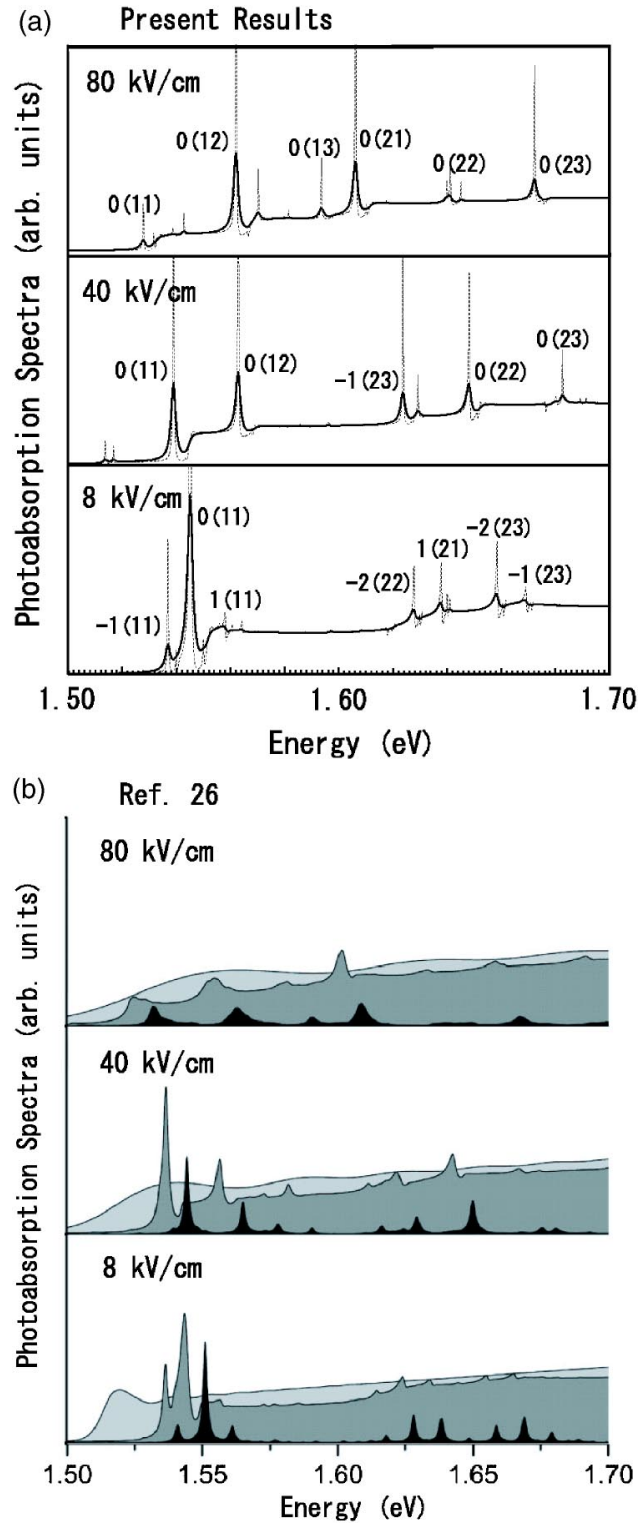

FIG. 8. The FR spectra of the $111 / 17-\AA \mathrm{GaAs} / \mathrm{Ga}_{0.7} \mathrm{Al}_{0.3} \mathrm{As}$ with $F=80,40$, and $8 \mathrm{kV} / \mathrm{cm}$ as a function of photon energy $E$. This system is the same as that of Ref. 26. (a) The calculated spectra with $N_{\text {site }}=20$ and with the Lorentzian broadening of $\Gamma$ $=2 \mathrm{meV}$ (the solid traces) and $0.3 \mathrm{meV}$ (the dotted traces). In the label of the $n\left(b_{e} b_{h}\right)$ state, the comma separating $b_{e}$ and $b_{h}$ is removed merely for typographical reasons; this should read as $n\left(b_{e}, b_{h}\right)$. Refer to the text for the meaning of this label. (b) The calculated spectra reported in Ref. 26, indicated by the traces with a dark gray area. In addition, the optical density of state and the spectra of the bulk GaAs are also shown by the traces with black and light-gray areas, respectively.

with each other. At $F=80 \mathrm{kV} / \mathrm{cm}$, where the Zener breakdown is considered significant, it is recognized that the onset of absorption is noticed to shift toward the lower-energy side below the energy $\varepsilon_{0(1,1)}$ to some extent in Figs. 8(a) and 8(b). This is due to the delocalization of the WSL subband wave function across several periods. The following text consists of a more detailed analysis of this effect, which is one of the purposes of the present paper.

\section{RESULTS AND DISCUSSION}

\section{A. Overview}

The calculated spectra of the 35/11-ML GaAs $/ \mathrm{Ga}_{0.75} \mathrm{Al}_{0.25} \mathrm{As}$ SLs with $F$ ranging widely from 10 to $200 \mathrm{kV} / \mathrm{cm}$ are shown in Fig. 9; the traces, represented by solid, dotted, and chain lines, indicate the FR spectra with coupled WSL channels, the FR spectra with noninteraction WSL channels (under the Kane approximation), and the free spectra without exciton effects [by setting $V$ to zero in Eq. (1)], respectively, using $N_{b}=6$ and $N_{\text {site }}=20$. Here, a peak of the exciton $1 s$ state supported by the channel $j$ is labeled as $j=n\left(b_{e}, b_{h}\right)$. Both these FR spectra, obtained by the full calculation and approximation, are manipulated by convoluting the original natural spectra by the Lorentzian function with $\Gamma=2 \mathrm{meV}$. The free spectra, however, remain unconvoluted and they have a stepped shape. The reference spectra obtained under the Kane approximation for the purpose of comparison are shown only in Fig. 9(a) for $F=10 \mathrm{kV} / \mathrm{cm}, 9$ (c) for $70 \mathrm{kV} / \mathrm{cm}, 9$ (f) for $100 \mathrm{kV} / \mathrm{cm}$, and 9(i) for $190 \mathrm{kV} / \mathrm{cm}$. As is seen in these figures, apart from minor changes, the spectra remain almost unaltered above $F=60 \mathrm{kV} / \mathrm{cm}$ with respect to the variance in $F$. This is because the Kane wave functions become localized in a single well, independent of the variance in $F{ }^{64}$ Therefore, the reference spectra have been omitted in the other panels of Fig. 9.

At $F=10 \mathrm{kV} / \mathrm{cm}$ [Fig. 9(a)], the spectra obtained by both the full calculation and the approximation are identical to each other and the Zener tunneling is observed to be negligibly weak. Although all spectra have not been shown here in the region from $F=10$ to $50 \mathrm{kV} / \mathrm{cm}$, apart from the relatively weaker transitions of $-1(1,1)$ and $-2(1,1)$, a strong absorption begins with the $0(1,1)$ transition. (However, this is not always the case in the much weaker bias region of $\Omega_{B}$ $<\Delta_{1}$, for instance, the one corresponding to that in Fig. 6, since the transitions with negative WSL indices are dominant.) In the region from $F=60$ to $80 \mathrm{kV} / \mathrm{cm}$ [Figs. 9(b)-9(d)], the FR spectral profiles are seen to be strongly modulated with respect to the variance in $F$, differing considerably from the FR spectra under the Kane approximation. It is noteworthy that when $F$ traverses the anticrossing at approximately $F=70 \mathrm{kV} / \mathrm{cm}$, the onset of absorption shifts toward the lower-energy side below $\varepsilon_{0(1,1)}$ and the absorption intensities in the higher-energy region are reduced, probably due to the Zener resonance. It is considered that the redshift of the absorption tail edge always accompanies the intensity reduction in the higher-energy region, as a result of the conservation rule of the optical oscillator strength; i.e., the sum rule. A similar tendency is also observed in the region from $F=90$ to $110 \mathrm{kV} / \mathrm{cm}$ [Figs. 9(e)-9(g)]. In particular, the redshift of the absorption tail edge manifests itself more noticeably in the vicinity of the anticrossing at $F=100 \mathrm{kV} / \mathrm{cm}$, whereas this phenomenon disappears at $F=90$ and $110 \mathrm{kV} / \mathrm{cm}$, which is slightly away from the anticrossing region; that is, the dominant absorption begins again with the $0(1,1)$ transition. Therefore, it is speculated that this effect arises due to the Zener resonance. As $F$ increases to $200 \mathrm{kV} / \mathrm{cm}$ [see Figs. 9(h)-9(j)], an energy splitting pertinent to the anticrossing becomes larger due to stronger cou- 

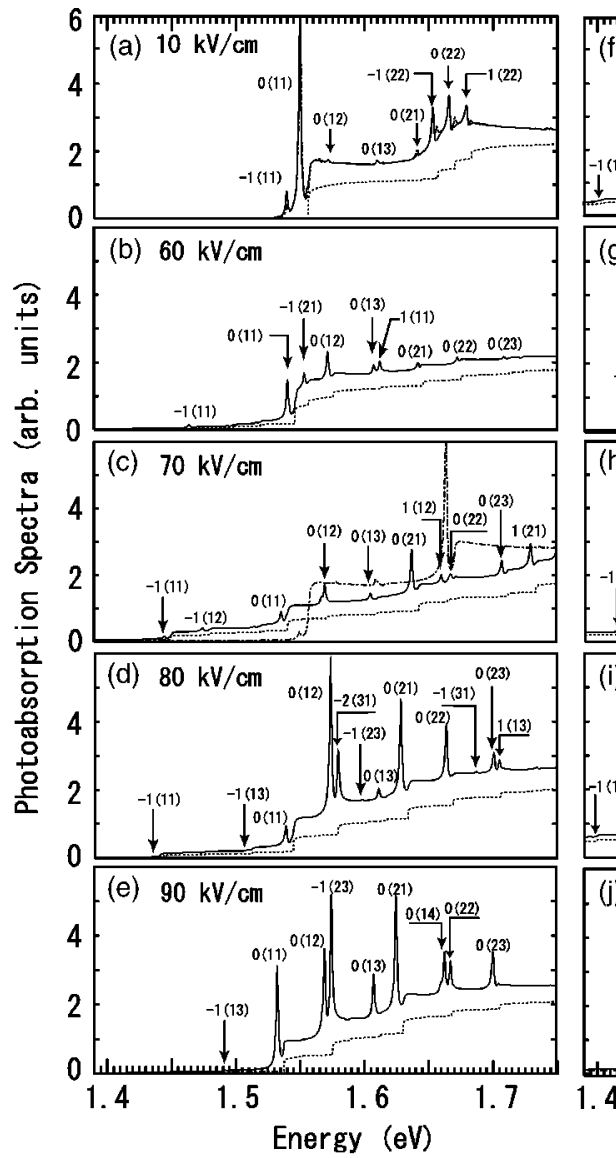
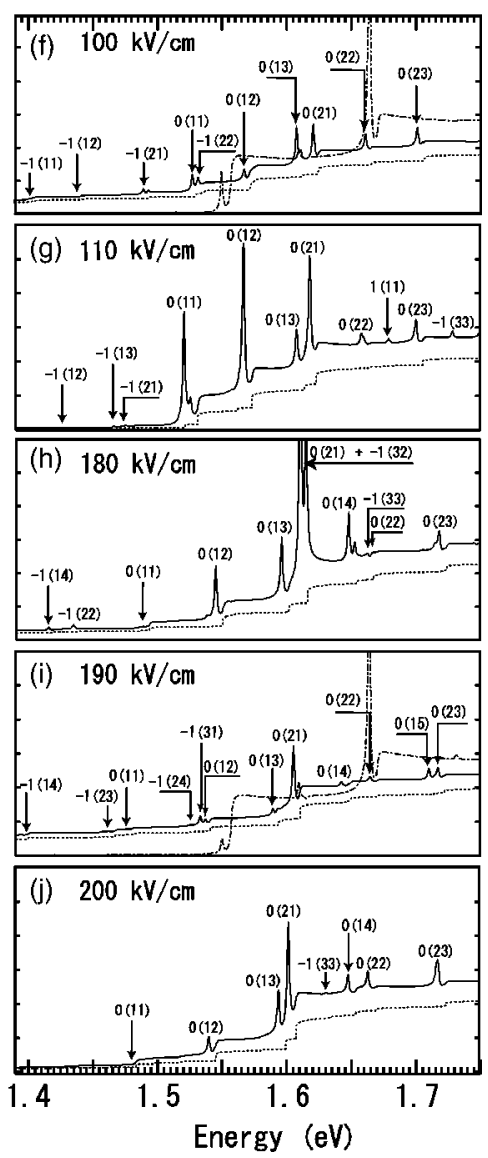

FIG. 9. The calculated FR spectra of the present WSL with $F=10-200 \mathrm{kV} / \mathrm{cm}$ are shown in panels (a)-(j) as a function of photon energy $E$. The solid traces are obtained from the full calculations, including $N_{b}=6, N_{\text {site }}=20$, and $N$ $=70-90$, while the dotted traces and the chain traces of panels (a), (c), (f), and (i) indicate the free spectra without exciton effects and the reference spectra given by the Kane approximation, respectively, for the purpose of comparison. The spectra indicated by the solid and chain lines are convoluted by the Lorentzian function with $\Gamma$ $=2 \mathrm{meV}$, while the free spectra are given with no convolution. In the label of the $n\left(b_{e} b_{h}\right)$ state, the comma separating $b_{e}$ and $b_{h}$ is removed for typographical reasons; this should read as $n\left(b_{e}, b_{h}\right)$. Refer to the text for the meaning of this label. plings among several WSL subband states, as is seen in Fig. 2(a). Similarly, the absorption edge is further shifted to the lower-energy side in the vicinity of the anticrossing at $F$ $=190 \mathrm{kV} / \mathrm{cm}$, although this effect is also observed to some extent at $F=180$ and $200 \mathrm{kV} / \mathrm{cm}$, differing from the spectra at $F=90$ and $110 \mathrm{kV} / \mathrm{cm}$. However, it is remarked that using $N_{b}=6$ to obtain the adopted WSL wave functions might not be sufficient to attain well-convergent results in such a large $F$ region.

To the best of our knowledge, mention to the redshift of the absorption tail edge was first made in Ref. 26. Nevertheless, the amount of this shift toward the lower-energy side below $\varepsilon_{0(1,1)}$ is very small, as is shown in the spectra at $F$ $=80 \mathrm{kV} / \mathrm{cm}$ in Fig. 8(b), compared with the spectra shown in Fig. 9(c). In particular, the significant role of anticrossings in conjunction with the Zener resonance has not been addressed thus far.

The following issues have been ascertained from the results of Fig. 9. First, the onset of absorption noticeably shifts toward the lower-energy side, accompanied by the reduction of absorption intensities in the higher-energy region above the tail. In other words, absorption strength is moved from the upper continuum to the lower-energy absorption tail. While tracing the changes in the reference free spectra of Fig. 9 (the dotted curves) in the energy region $E<\varepsilon_{0(1,1)}$ with respect to $F$, it is observed that the modulation pattern is in harmony with that of the FR spectra under consideration (the solid curves). Second, the FR peak intensity and position for the transitions in the energy region $E>\varepsilon_{0(1,1)}$ are noticeably modulated with respect to the variance in $F$. A discussion on the first issue is presented in Sec. III B, followed by another discussion on the second issue in Sec. III C.

\section{B. Redshift of the absorption edge}

Figure 10 shows the change in the absorption intensity at the photon energy of $E=1.47 \mathrm{eV}$ as a function of $F$ from 10 to $112 \mathrm{kV} / \mathrm{cm}$, where the result obtained by the full calculation of the FR spectra is indicated by filled circles that are connected by solid lines in order to aid the presentation. The open circles, filled squares, and open squares represent the other results obtained by model calculations, to be explained later, for the purpose of comparison with the result concerned and for examining the pattern of variance in the absorption intensity in further detail. It is evident that the intensity of the tail region of the spectra changes in a complex manner accompanying oscillations rather than in a monotonic manner as a whole; this tendency stands out in Figs. 10(b) and 10(c).

As seen in Fig. 10(c), there are three maxima of intensity at the positions of $F=95,100$, and $104 \mathrm{kV} / \mathrm{cm}$ (see the filled circles). Hereafter, the three biases in the ascending order are denoted as $F_{1}, F_{2}$, and $F_{3}$ for the sake of convenience. These biases coincide perfectly with each location of the anticrossing shown by the dashed circle in Fig. 2(b). Therefore, this observation leads to the speculation that the Zener resonance plays a decisive role in causing the redshift of the absorption tail edge. Moreover, this effect is quite localized in the prox- 


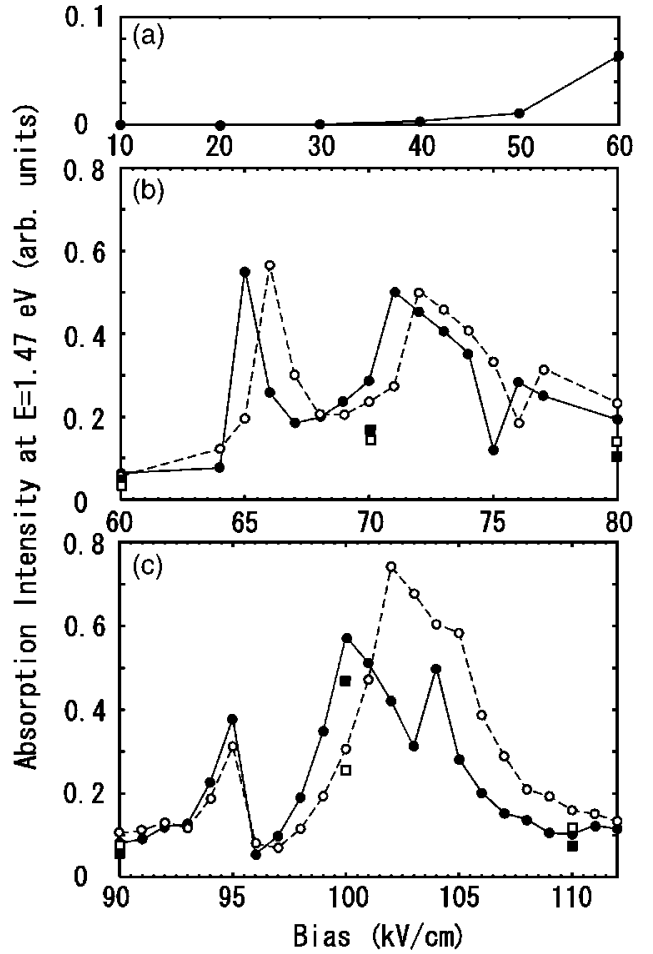

FIG. 10. Variance of the spectral intensity at $E=1.47 \mathrm{eV}$ in the present WSL as a function of $F$ in the regions where (a) the Kane approximation is still almost valid, (b) the anticrossing in the vicinity of $F=70 \mathrm{kV} / \mathrm{cm}$ is dominant, and (c) the anticrossing in the vicinity of $F=100 \mathrm{kV} / \mathrm{cm}$ is dominant. Here, the filled and open circles represent the FR spectra obtained by the full calculations and the model calculations, respectively. Refer to the text for the details on the calculations of the latter. These circles are connected by solid and dashed lines in order to aid the presentation. Moreover, the filled and open squares represent the free spectra without exciton effects, corresponding to the full and model calculations indicated by the filled and open circles, respectively.

imity of each anticrossing. For instance, the first peak begins at $F=93 \mathrm{kV} / \mathrm{cm}$ and ends at $F=96 \mathrm{kV} / \mathrm{cm}$, followed by the onset of the next rise. This succession of the localized peaks and dips relevant to the anticrossings are thus understood to give rise to the appearance of the oscillation structure in the intensity. On the basis of the two-channel Landau-Zener model applied to the anticrossing formation by channels of, say, $a$ and $b$, with diabatic energies of, say, $\epsilon_{a}(F)$ and $\epsilon_{b}(F)$, an effective range of the anticrossing, denoted by $\delta F_{c}$, is given by $\delta F_{c}=4 v_{c} / \alpha_{c}$ at $F=F_{c}(c=1-3){ }^{65}$ In this case, $v_{c}$ implies the strength of the coupling between $a$ and $b$, and $\alpha_{c}=|d \delta \epsilon / d F|_{F=F_{c}}$, where $\delta \epsilon=\epsilon_{b}(F)-\epsilon_{a}(F)$. Employing Eq. (18) for $\epsilon_{i}(F)(i=a, b), \alpha_{c}$ is approximated by $\alpha_{c} \approx\left|\delta n_{c}\right| d$, where $\delta n_{c}$ is the difference of the WSL indices of these two channels. In actuality, $\delta n_{1} \approx 4$ between $a=0(1)$ and $b=4(4)$, $\delta n_{2}=2$ between $0(1)$ and 2(3), $\delta n_{3}=3$ between 1(2) and 4(4), and $v_{1} \approx v_{3}<v_{2}$ at an estimate from the magnitude of the energy splitting due to the anticrossing at $F=F_{c}$ in Fig. 2(b). Hence, this leads to an estimation that $\delta F_{2}$ is greater than $\delta F_{1}$ and $\delta F_{3}$, which is in good conformity with the oscillation structure seen in Fig. 10(c). Additionally, it is also remarked that the intensity almost vanishes away from the an- ticrossing region that comprises these three main anticrossings; i.e., it vanishes at approximately $F=90$ and $110 \mathrm{kV} / \mathrm{cm}$.

A similar tendency of the oscillation is also observed in Fig. 10(b), where the anticrossings at $F=65,71$, and $76 \mathrm{kV} / \mathrm{cm}$ are likely to dominate the intensities of the absorption tail. In contrast, in Fig. 10(a) for the smaller bias regime, a minute portion of the redshift of the absorption tail edge is observed at $E=1.47 \mathrm{eV}$. Furthermore, the intensity only increases monotonically with an increase in $F$, without resulting in an oscillating structure. The increase is despite the fact that there are a large number of the anticrossings in this region, as seen in Fig. 2(a), which intertwine as many pairs of subband states with each other in a complex manner, but with negligibly weak couplings. The same traces as those observed in Fig. 10 for the change in the absorption intensity are obtained also at the lower photon energy of $E=1.42 \mathrm{eV}$, though not shown here. According to this observation, the intensity of the absorption tail is reduced to a larger extent in the region of $F=60-80 \mathrm{kV} / \mathrm{cm}$ as compared with that observed in Fig. 10(b), whereas the spectral intensity and alteration in the other regions are similar to those in Figs. 10(a) and 10(c). This implies that the absorption tail does not extend down to such a lower-energy side yet for $F$ $=60-80 \mathrm{kV} / \mathrm{cm}$. Thus, the degree of the redshift of the absorption tail edge depends on the magnitude of $F$ causing the delocalization of WSL subband wave functions.

As mentioned in Sec. III A, the modulation pattern of the reference free spectra of Fig. 9 (the dotted curves) in the energy region $E<\varepsilon_{0(1,1)}$, with respect to $F$, is qualitatively in good accordance with that of the FR spectra under consideration (the solid curves). This allows us to qualitatively observe the concerned effect of the redshift based on the free spectra. The mathematical formulation of the spectra required here is mentioned in Appendix C. As shown in Eq. (C9), the dipole moment of the noninteracting $e-h$ pair, $\mu_{\alpha}^{0}(E)$, is essentially governed by two factors $-w_{j}^{0}$ and $U_{j \alpha} C_{\alpha}$ - where the former is the magnitude of overlap between the wave functions of $e$ and $h$ belonging to the $j$ th subband and the latter is a product of a normalization constant of the in-plane radial wave function of the $\alpha$ th channel $C_{\alpha}$ and a unitary matrix $U_{j \alpha}$. This unitary matrix determines the interchannel couplings between $j$ and $\alpha$ through the mixing of the reduced mass $m_{\|}$in the region of the confining potential well with that in the region of the potential barrier. The eigenvalues $\lambda_{\alpha}$ of Eq. (C6) vary from 22.83 to 23.82 at $F=100 \mathrm{kV} / \mathrm{cm}$; note that the inverse of the reduced mass $1 / m_{\|}$in the well region, hereafter denoted as $1 / m_{\|}^{0}$, equals 23.75. According to these calculations, for a certain channel $\alpha$ whose eigenvalue $\lambda_{\alpha}$ deviates considerably from $1 / m_{\|}^{0}$, the diagonal element $U_{\alpha \alpha}$ is drastically reduced to less than 0.5 , and instead, the off-diagonal elements $\left|U_{j \alpha}\right|(j \neq \alpha)$ are enhanced. This causes the interchannel coupling between $j$ and $\alpha$.

Let the interchannel coupling effect due to $U_{j \alpha} C_{\alpha}$ be examined in the following by separating it from the concerned FR spectra, ${ }^{66}$ though both effects due to $w_{j}^{0}$ and $U_{j \alpha} C_{\alpha}$ are essentially attributed to the delocalization of WSL subband wave functions across several periods. In order to examine 
this coupling effect, the model calculations of the FR spectra are implemented by artificially replacing the inverse of the reduced mass $1 / m_{\|}$in the barrier region by $1 / m_{\|}^{0}$. Such a manipulation brings $U_{j \alpha} C_{\alpha}$ to $\delta_{j \alpha} \sqrt{m_{\|}^{0}}$, leading to $\mu_{\alpha}^{0}(E)$ $\approx w_{\alpha}^{0} \sqrt{m_{\|}^{0}}$ in Eq. (C9). In order to make this treatment theoretically consistent, the inverse of the axial mass $1 / m_{z}^{(e)}$ $\left(1 / m_{z}^{(h)}\right)$, although it is a function of $z_{e}\left(z_{h}\right)$, is also replaced by a constant value, for example, $1 / m_{z}^{(e) 0}\left(1 / m_{z}^{(h) 0}\right)$, which is equal to the inverse of the axial mass in the well region. The absorption intensities of the FR spectra thus modeled are shown in Figs. 10(b) and 10(c) by the open circles; these are connected by dashed lines to aid the presentation. Hereafter, the modeled spectra are termed as spectra B, while the spectra represented by the filled circles are termed as spectra A. In Figs. 10(b) and 10(c), the results of the free spectra without exciton effects (depicted in Fig. 9) are also represented by the filled squares for some values of $F$ along with those corresponding to spectra B with $1 / m_{\|}=1 / m_{\|}^{0}, 1 / m_{z}^{(e)}$ $=1 / m_{z}^{(e) 0}$, and $1 / m_{z}^{(h)}=1 / m_{z}^{(h) 0}$, which are shown by the open squares. The variance of spectra A and B with respect to $F$ is seen to be qualitatively consistent with that of the corresponding free spectra. Therefore, the interchannel coupling effect due to the reduced-mass mixing through $U_{j \alpha} C_{\alpha}$ would be extracted by comparing spectra A with spectra B.

In order to ensure the validity of this statement, an effect of the replacement of $1 / \mathrm{m}^{(k)}$ in the barrier region of the confining potential by $1 / m_{z}^{(k) 0}$ should be checked. According to our calculations, this replacement results in an increase in the WSL subband energies $\epsilon_{j_{k}}^{(k)}$ of $k=e$ and $h$ by the order of $1 \times 10^{-4} \mathrm{Ry}$ and $1 \times 10^{-5} \mathrm{Ry}$, respectively; for instance, at $F=100 \mathrm{kV} / \mathrm{cm}, \epsilon_{0(1)}^{(e)}$ changes from $-3.123 \times 10^{-3} \mathrm{Ry}$ to $-2.959 \times 10^{-3} \mathrm{Ry}$, and $\epsilon_{0(1)}^{(h)}$ changes from $3.993 \times 10^{-3}$ Ry to $4.021 \times 10^{-3}$ Ry. Just in such a slight degree as the subband energies are changed, the locations of anticrossings seen in Figs. 2 and 4 are modified. Similarly, the WSL wave functions of $\phi_{j_{k}}^{(k)}$ remain almost unaltered, as shown by the chain lines in Figs. 3 and 5, causing minute changes in the strength of couplings between the SL minibands for anticrossings. Therefore, since $w_{j}^{0}$ is maintained approximately invariant, the difference between spectra A and B can be considered to be exclusively attributed to the reduced-mass mixing effect.

In Fig. 10(c), there is a marked difference in the intensity and pattern of variance between spectra $\mathrm{A}$ and $\mathrm{B}$ in the primary anticrossing region of $F=95-110 \mathrm{kV} / \mathrm{cm}$. However, they are almost identical when they are observed further from this region. In particular, the discrepancy looks considerably large around the region of $F=100 \mathrm{kV} / \mathrm{cm}$. Figure 11 shows spectra A (the solid traces) and spectra B (the dotted traces) in the wide $E$ region at $F=90,100$, and $110 \mathrm{kV} / \mathrm{cm}$, indicating that both spectra are almost identical except in $E$ $<\varepsilon_{0(1,1)}$ in Fig. 11(b); however, slight shifts of the exciton peak positions are observed, in particular, in Fig. 11(c). Hence, it is likely that the reduced-mass mixing manifests itself in the lower $E$ side when Zener tunneling is relatively strong. ${ }^{67}$ On the other hand, in Fig. 10(b) for the smaller $F \mathrm{~s}$ resulting in weaker Zener tunneling, spectra $\mathrm{B}$ are almost identical to spectra A both in intensity and variance pattern;

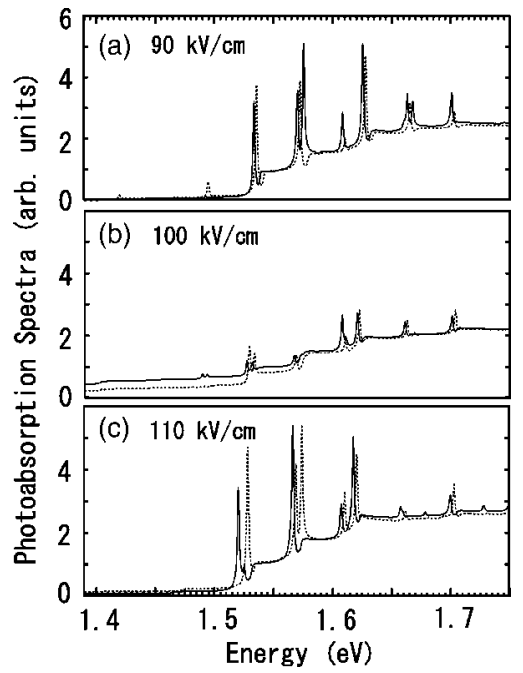

FIG. 11. The calculated FR spectra of the present WSL with $F$ =(a) $90 \mathrm{kV} / \mathrm{cm}$, (b) $100 \mathrm{kV} / \mathrm{cm}$, and (c) $110 \mathrm{kV} / \mathrm{cm}$ as a function of photon energy $E$, where the Lorentzian convolution is used with $\Gamma=2 \mathrm{meV}$. The spectra indicated by the solid lines are the same as those in Figs. 9(e)-9(g), while those represented by the dotted lines are the results obtained by the corresponding model calculations. Refer to the text for the details on the calculations of the latter.

however, there is a small overall shift of $F$ by $1 \mathrm{kV} / \mathrm{cm}$ that renders the observation of any definite physical meaning difficult. Consequently, the reduced-mass mixing seems minor in this anticrossing region as compared with that in Fig. 10(c).

\section{Spectral modulation}

As can be observed from Fig. 9, the Zener tunneling causes the spectral modulation of the FR spectra as well as the abovementioned redshift of the absorption tail edge. In order to trace the modulation pattern in more detail, Fig. 12 shows the spectra in the vicinity of the arrowed peak positions of the $0(1,1), 0(1,2), 0(2,2), 0(1,3)$, and $0(2,1)$ states for $F=10-110 \mathrm{kV} / \mathrm{cm}$, with the Lorentzian broadening of $\Gamma$ $=2 \mathrm{meV}$ (solid lines) along with that of $\Gamma=0.3 \mathrm{meV}$ (dotted lines) to indicate shapes of the corresponding natural spectra. In every state, both the peak position and height vary in an irregular manner without displaying monotonic changes. These irregular changes manifest themselves at approximately $F=70$ and $100 \mathrm{kV} / \mathrm{cm}$, in particular, possibly due to the formation of anticrossings between the electron subbands $1-2$ and $1-3$, respectively. Thus, it is speculated that these FR spectra are greatly modulated by the Zener resonance because of the anomalous changes in the associated subband wave functions. Moreover, apart from such irregularity, the spectral intensities of the vertical transitions of $0(1,2), 0(1,3)$, and $0(2,1)$ with different miniband indices that $b_{e} \neq b_{h}$ are enhanced with an increase in $F$, as is shown in Figs. 12(b), 12(d), and 12(e). The enhancement arises exclusively from the Zener breakdown. This is evident from Figs. 9(a), 9(c), 9(f), and 9(i) by comparing the spectra obtained by the full calculations (the solid traces) with the approximate ones (the chain traces). In actuality, the Kane approximation makes 

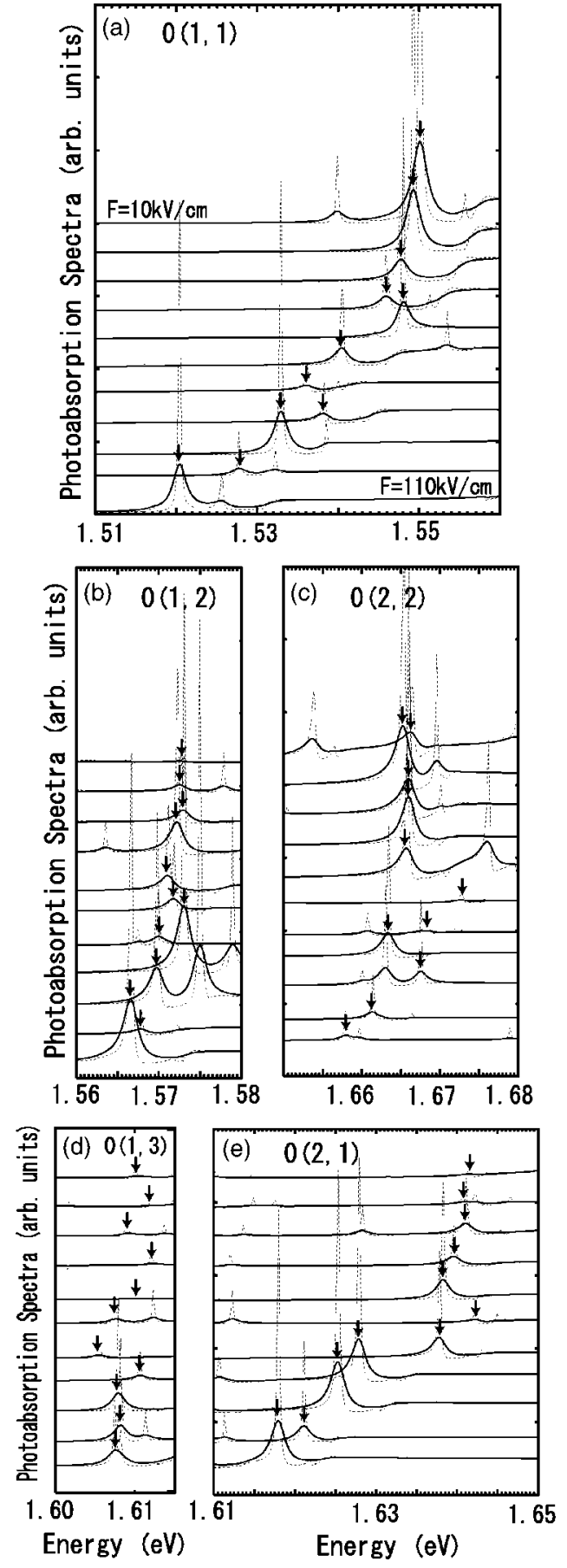

FIG. 12. The FR spectra of the present WSL in the vicinity of the $1 s$ state pertaining to (a) $0(1,1),($ b) $0(1,2),($ c) $0(2,2),(d) 0(1,3)$, and (e) $0(2,1)$. In each panel, the traces, which are shifted vertically to enhance the presentation, represent the spectra at $F$ $=10-110 \mathrm{kV} / \mathrm{cm}$ in an ascending order of $F$ from the top to the bottom. The peak positions of the concerned FR states are indicated by arrows. The spectra with the Lorentzian broadening of $\Gamma=2$ and $0.3 \mathrm{meV}$ are represented by the solid and dotted lines, respectively.

these intensities negligible for large $F$ because both the approximated subband wave functions of $e$ and $h$, which are mostly confined in the region $0 \leqslant z_{e} \leqslant d$ and $0 \leqslant z_{h} \leqslant d$, respectively, have different nodal structures and hence an overlap between these wave functions is reduced. Further, as seen in Figs. 12(a) and 12(e), the peak positions of $0(1,1)$ and $0(2,1)$ exhibit a more pronounced shift to the lower-energy side, when compared with those of $0(1,2)$ and $0(2,2)$, where the shift is very slight. This tendency is presumably due to large modification of the wave function of $b_{h}=1$ for a large $F$ as compared with that of $b_{e}=1$ and 2 [see also Fig. 5(a)]. Finally, we refer to the linewidth broadening due to the Zener breakdown. In order to extract this from the exciton spectra, the natural width (FR width modified by the Zener tunneling) of a concerned spectrum should be much greater than the homogeneous broadening of $\Gamma$; hence, high-resolution calculations are indispensable for it. It would be expected from the results of the spectrum of the $0(1,1)$ state in Ref. 27 , that the stronger Zener breakdown broadens the spectral width. However, as far as the $F \leqslant 110 \mathrm{kV} / \mathrm{cm}$ region is concerned, the linewidth broadening is not pronounced yet for every FR spectrum of Fig. 12. One of the possible reasons for this might be that the SLs employed in Ref. 27 ( $x$ $=0.08$ ) are so shallow that the Zener tunneling affects the spectral width to a greater extent than the SLs under consideration here. This issue will be deferred to a subsequent paper.

The $0(1,1), 0(2,2)$, and $0(1,3)$ transitions will be discussed in further detailed. As already discussed in Ref. 29, the absorption spectra of the $0(1,1)$ state rapidly disappear due to the field-induced delocalization with an increase in $F$ to $40 \mathrm{kV} / \mathrm{cm}$; however, this is not the case for larger values of $F$ as stated above. Figure 12(a) also indicates the monotonic redshift akin to the character of the quantum-confined Stark effect. ${ }^{68,69}$ Furthermore, in the reported experiment, ${ }^{41}$ the change of Fano's $q$-value in the FR spectra with respect to $F$ was measured to approximately $30 \mathrm{kV} / \mathrm{cm}$. This indicates that by increasing $F,|q|$ increases monotonically without a change in its sign; i.e., the asymmetric FR spectra become symmetric. This was conducted for relatively weak $F$ fields up to a maximum of approximately $30 \mathrm{kV} / \mathrm{cm}$, where the Zener tunneling is still insignificant. Indeed, in Fig. 12(a), the spectra for $F=10-40 \mathrm{kV} / \mathrm{cm}$ with strong peaks and $q$ $<0$ appear to follow the monotonic change similar to this experiment. However, the spectrum exhibits an anomalous change in $q$ above this; in particular, $q$ appears positive at $F=50 \mathrm{kV} / \mathrm{cm}$, and can be brought back to a negative value at $F=60-80 \mathrm{kV} / \mathrm{cm}$. In this figure, this is more clearly seen in the dotted curves than in the solid ones.

It is likely that the $0(2,2)$ state provides the most dominant spectrum for relatively weak biases because of a vertical transition from the $h$ subband to the $e$ subband with the same miniband index, apart from the abovementioned $0(1,1)$ state. As seen in Fig. 12(c), the pattern of changes in the spectral height and the $q$ value for $F=10-50 \mathrm{kV} / \mathrm{cm}$ is similar to that of the $0(1,1)$ state. However, the peak abruptly disappears at $F=60$ and $70 \mathrm{kV} / \mathrm{cm}$, and is retrieved with $|q| \gg 1$ at $F$ $=80 \mathrm{kV} / \mathrm{cm}$. The spectral intensity is noticeably reduced by increasing $F$ to more than $90 \mathrm{kV} / \mathrm{cm}$. Small modifications in the spectral profile are also noticed due to an overlapping resonance with the adjacent FR spectra. ${ }^{50,63,69}$ It is evident that these patterns of spectral modulation shown here are very different from those obtained in the abovementioned experiment. $^{41}$

As regards the FR spectra pertaining to $0(1,3)$, the spectral profile covering the entire Fig. 12(d) is hard to discern 


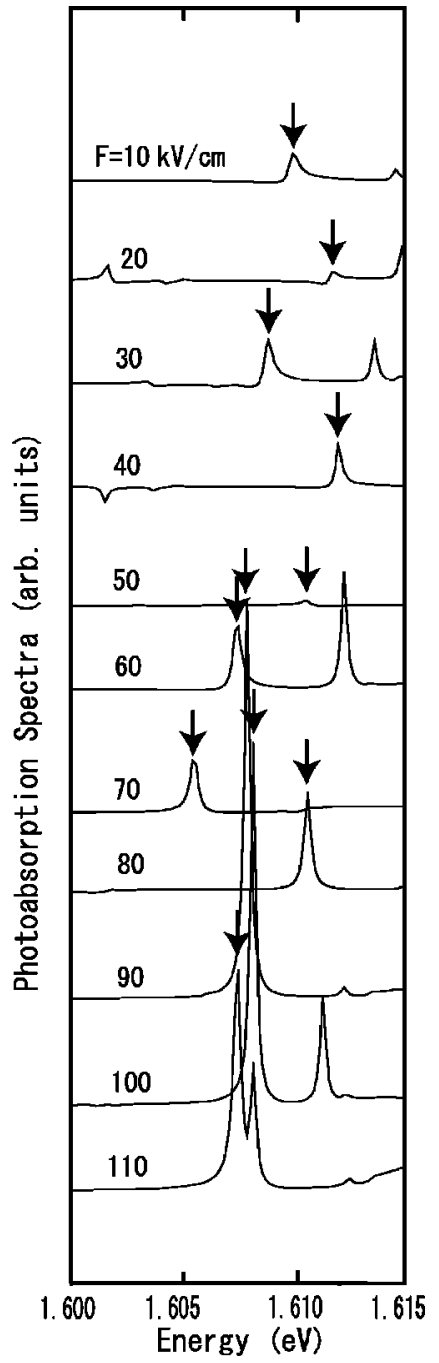

FIG. 13. Expanded view of the same spectra as those with $\Gamma$ $=0.3 \mathrm{meV}$ of Fig. 12 (d) for the state pertaining to $0(1,3)$.

because of its small intensity. Hence, the expanded spectra of the dotted traces in this figure are depicted in Fig. 13. Although most excitonic FR spectra usually have a negative Fano's $q$ value, it is known that the $0(1,3)$ state exhibits a characteristic spectral profile with a positive $q$-value in quasi-two-dimensional semiconductor heterostructures, i.e., a spectral dip followed by a peak, ${ }^{70,71}$ and this profile was observed in the experiment. ${ }^{72}$ As is seen in Fig. 13, the spectral profile is gradually altered by retaining $q>0$ for $F$ $=10-40 \mathrm{kV} / \mathrm{cm}$, followed by a disappearance at $F$ $=50 \mathrm{kV} / \mathrm{cm}$. Similar to the modulation pattern of Fig. 12(a), the spectra are retrieved with $|q| \gg 1$ and $q<0$, and with a large intensity at $F=90 \mathrm{kV} / \mathrm{cm}$. Nevertheless, it would be difficult to confirm such a subtle modulation of this spectral inversion by experiments. In fact, in Fig. 12(d), the modulation pattern looks smeared by the external broadening mechanism with $\Gamma=2 \mathrm{meV}$.

\section{SUMMARY}

Optical absorption spectra of a WSL exciton under a strong $F$ field along the direction of crystal growth are com- prehensively examined by solving the multichannel scattering problem for the concerned FR. First, the validity and feasibility of the adopted method have been initially confirmed by applying it to the already reported systems for WSL excitons, followed by a comparison between them. Next, the FR spectra of the concerned coupled WSL system have been calculated in order to explore the modulation pattern of these spectra. It is concluded that through delocalization of the subband wave functions Zener tunneling plays the key role of an anomalous variance of the FR spectra with respect to $F$. In particular, the spectral modulation is clearly visible when a series of WSL subband states are energetically aligned with each other, resulting in anticrossings with strong repulsion due to Zener resonance. The Zener resonance is observed to cause a notable shift in the absorption edge toward the lower energy side. However, this effect is suppressed by a slight variance in $F$ away from the anticrossing region. In the $F=90-112 \mathrm{kV} / \mathrm{cm}$ region, in particular, the intensities of these tail spectra in the $E<\varepsilon_{0(1,1)}$ region change in an oscillating manner with respect to $F$. Indeed, the locations of the anticrossings are precisely reflected on this pattern of variance. According to the model calculations, the interchannel coupling effect due to the reduced-mass mixing as well as the overlap effect between the subband wave functions of $e$ and $h$ contributes to the enhancement of the absorption tail spectra. In addition to this unusual phenomenon, it is also shown that the peak positions, heights, and $q$ values of the FR spectra in the $E>\varepsilon_{0(1,1)}$ region are modulated in an irregular manner due to the Zener breakdown as $F$ traverses the anticrossing region. For instance, the spectra for the $1 s$ state pertaining to the $0(2,2)$ subband disappear abruptly at a certain bias, followed by the recovery of the intensities out of this region. Moreover, the spectra of the $0(1,1)$ and $0(1,3)$ exhibit a profile inversion from a positive Fano $q$ value to a negative one. This is in contrast with the existing experiment for weak $F$ fields, where the $q$ value varies monotonically.

\section{ACKNOWLEDGEMENTS}

This research was financially supported by Grant-in-Aid for Scientific Research (B) and Grant-in-Aid for Scientific Research (C) from the Japan Society for the Promotion of Science.

\section{APPENDIX A: METHOD TO SOLVE EQ. (16)}

The numerical procedure of obtaining a set of WSL wave functions $\left\{\phi_{j_{k}}^{(k)}\right\}$ is presented, where $k=e, h$. In particular, the first stage (i) of the stepwise procedure provided in Sec. II A is explained in detail. For convenience of presentation, the Schrödinger equation for the particle of $k$ in a single quantum well is initially expressed as

$$
\left(T+v_{k}(z)-e_{i}^{(k)}\right) \xi_{i}^{(k)}(z)=0
$$

where 


$$
T=-\frac{d}{d z}\left(\frac{1}{2 m_{z}^{(k)}}\right) \frac{d}{d z},
$$

$v_{k}$ is the confining potential with a barrier height of $v_{k}^{0}$, and $e_{i}^{(k)}$ is the $i$ th energy associated with the eigenfunction $\xi_{i}^{(k)}$.

Next, we introduce the following equation for a set of Sturmian functions $\left\{\eta_{i}^{(k)}\right\} .53,54$

$$
\left[T+\alpha_{i} w_{k}(z)-\beta_{k}\right] \eta_{i}^{(k)}(z)=0,
$$

where $w_{k}(z)=v_{k}-v_{k}^{0}$ and $\beta_{k}=e_{1}^{(k)}-v_{k}^{0}$; here $e_{1}^{(k)}$ is the lowest eigenvalue of Eq. (A1). Moreover $\alpha_{i}$ is the $i$ th eigenvalue associated with $\eta_{i}^{(k)}$. The set of Sturmian functions are square-integrable since the associated eigenvalues $\left\{\alpha_{i}\right\}$ are always discrete and $\alpha_{i} \geqslant 1$. This feature distinguishes from the set of wave functions of Eq. (A1) that are not squareintegrable for the continuum spectra. It is also evident that $\eta_{1}^{(k)}$ coincides with $\xi_{1}^{(k)}$ for $\alpha_{1}=1$. The Sturmian functions are normalized by the following equation:

$$
\int\left[\eta_{i}^{(k)}\right]^{*} w_{k}(z) \eta_{j}^{(k)} d z=\delta_{i j}
$$

The functions are calculated using an expansion with respect to a set of B-spline functions $\left\{B_{l \kappa}(z)\right\}$ as follows: ${ }^{51}$

$$
\eta_{i}^{(k)}(z)=\sum_{l} B_{l \kappa}(z) \mathcal{X}_{l i}^{(k)}
$$

where $B_{l \kappa}(z)$ is the $l$ th normalized B-spline of an order $\kappa$ with a knot sequence $\left\{z_{l}\right\}$. Following the BSCM, ${ }^{52}$ this problem can be formulated into a standard algebraic equation for obtaining a set of coefficients $\left\{\mathcal{X}_{l i}^{(k)}\right\}$, by imposing vanishing boundary conditions on $\eta_{i}^{(k)}(z)$. Some advantages of adopting the Sturmian basis set and the BSCM have already been discussed in Sec. II A.

Once the set of the Sturmian functions is obtained, a wave function $\zeta_{K B_{k}}^{(k)}(z)$ of the SLs is expanded as follows:

$$
\zeta_{K B_{k}}^{(k)}(z)=\frac{1}{\sqrt{N_{\text {site }}}} \sum_{L} e^{i K L d} W_{B_{k}}(z-L d),
$$

where $K$ and $B_{k}$ are the Bloch momentum and a miniband index, respectively, and the Wannier function is expressed as

$$
W_{B_{k}}(z)=\sum_{j} \eta_{j}^{(k)}(z) \mathcal{Y}_{j B_{k}}
$$

A set of coefficients $\left\{\mathcal{Y}_{j B_{k}}\right\}$ is obtained by introducing Eq. (A6) in the Schrödinger equation relevant to the SL Hamiltonian. Equation (16) can be solved by further expanding the WSL wave function $\phi_{j_{k}}^{(k)}$ in terms of the set of the SL wave functions $\left\{\zeta_{K B_{k}}^{(k)}\right\}$ as follows:

$$
\phi_{j_{k}}^{(k)}(z)=\sum_{K, B_{k}} \zeta_{K B_{k}}^{(k)}(z) \mathcal{Z}_{K B_{k} j_{k}}
$$

where $\left\{\mathcal{Z}_{K B_{k^{j}} j_{k}}\right\}$ is a set of expansion coefficients that are to be determined.

\section{APPENDIX B: VERIFICATION OF NUMERICAL CONVERGENCE OF THE WSL ENERGIES AND WAVE FUNCTIONS}

The most standard method of verifying the numerical accuracy of calculated eigenenergies and eigenfunctions is to trace the changes of these quantities with respect to a size of the basis set employed for the computations. As is well known, the eigenvalues calculated on the basis of the Rayleigh-Ritz variational principle ${ }^{61}$ decrease monotonically with an increase in the size of the basis set, eventually reaching the convergence. In this case, the numerical accuracy of the electron WSL energies $\epsilon_{j_{e}}$ and the associated wave functions $\phi_{j_{e}}$, which are obtained with $N_{b}=6$ and $N_{\text {site }}=20$ for $j_{e}=0\left(b_{e}\right)\left(b_{e}=1-5\right)$, has been confirmed by changing the number of the included minibands $N_{b}$ from 1 to 5 with fixed $N_{\text {site }}$. In Table II, the calculated results are listed for $F=90,100$, and $110 \mathrm{kV} / \mathrm{cm}$, where the transition at $F=100 \mathrm{kV} / \mathrm{cm}$ is due to the resonant Zener tunneling, and the other two transitions are due to the nonresonant Zener tunneling, as is seen in Fig. 2. The energies provided with $N_{b}=1$ are considered to be only those under the Kane approximation, and the results thus approximated for $b_{e} \geqslant 2$ do not ensure the validity of the Rayleigh-Ritz variational principle. The basis set with $N_{b}=6$ permit us to obtain an accuracy for the coupled WSL subband energies up to at least the fourth, third, and second digits of the significant figure for $b_{e}=1-2,3-4$, and 5, respectively. Thus, the convergence of the energies with such a small size of the basis set proves to be excellent. Figure 14 shows the convergence patterns of the associated wave functions with respect to $N_{b}$, which are represented by $\mathrm{WF}-N_{b}$ based on the notation defined in Sec. II A. Here, the solid line, the dotted line, and the chain line represent the results of calculations with $N_{b}=6,5$, and 4, respectively, and with $N_{s i z e}=20$. For $b_{e} \leqslant 3$ in particular, it is quite difficult to discern the difference of the results of WF-6 from those of WF-4 and WF-5 from this figure. Therefore, the excellent convergence of WF-6 is reverified.

\section{APPENDIX C: FREE SPECTRA}

The Wannier equation relevant to the free spectra is expressed as

$$
\left(H_{0}-E\right) \Psi^{0}(|\rho|, \Omega)=0
$$

where

$$
\begin{aligned}
H_{0}\left(\rho, z_{e}, z_{h}\right) & =H_{e x}\left(\rho, z_{e}, z_{h}\right)-V\left(\rho, z_{e}-z_{h}\right) \\
& =-\frac{1}{2 m_{\|}} \nabla_{\rho}^{2}+h_{w s l}\left(z_{e}, z_{h}\right) .
\end{aligned}
$$

The wave function $\Psi^{0}$ is expanded with respect to the basis set $\left\{\varphi_{j}\right\}$ from Eq. (13) as

$$
\Psi_{\alpha}^{0}(|\rho|, \Omega)=\frac{1}{\sqrt{|\rho|}} \sum_{j} \varphi_{j}(\Omega) f_{j \alpha}^{E}(|\rho|),
$$

where the adiabatic channel function $\Phi_{\mu}$ from Eq. (6) coincides with $\varphi_{j}$ when $V=0$, or, equivalently, when $|\rho| \rightarrow \infty$, by 
TABLE II. Variance of the energies $\epsilon_{0\left(b_{e}\right)}$ of the electron WSL subband states expressed in the unit of $10^{-2} \times$ Ry with respect to the total number of minibands included in the calculations $N_{b}$ at $F=$ (a) 90 , (b) 100 , and (c) $110 \mathrm{kV} / \mathrm{cm}$. Note that the resonant Zener tunneling is dominant at $F=100 \mathrm{kV} / \mathrm{cm}$ while the nonresonant Zener tunneling is dominant at $F=90$ and $110 \mathrm{kV} / \mathrm{cm}$. Refer to the text for further detail.

\begin{tabular}{|c|c|c|c|c|c|c|}
\hline & $b_{e}=1$ & $b_{e}=2$ & $b_{e}=3$ & $b_{e}=4$ & $b_{e}=5$ & $b_{e}=6$ \\
\hline \multicolumn{7}{|c|}{ (a) $F=90 \mathrm{kV} / \mathrm{cm}$} \\
\hline$N_{b}=1$ & -0.204317 & 0.466814 & 1.62776 & 3.39729 & 5.92929 & 9.24860 \\
\hline$N_{b}=4$ & -0.237256 & 0.442686 & 1.74509 & 3.33703 & & \\
\hline$N_{b}=5$ & -0.237343 & 0.442572 & 1.73449 & 3.32202 & 5.95510 & \\
\hline$N_{b}=6$ & -0.237348 & 0.442570 & 1.73304 & 3.31951 & 5.94449 & 9.26318 \\
\hline \multicolumn{7}{|c|}{ (b) $F=100 \mathrm{kV} / \mathrm{cm}$} \\
\hline$N_{b}=1$ & -0.252157 & 0.418974 & 1.57992 & 3.34945 & 5.88145 & 9.20076 \\
\hline$N_{b}=4$ & -0.309658 & 0.381790 & 1.65020 & 3.37386 & & \\
\hline$N_{b}=5$ & -0.312283 & 0.380782 & 1.64726 & 3.35039 & 5.91149 & \\
\hline$N_{b}=6$ & -0.312334 & 0.380733 & 1.64721 & 3.34989 & 5.89168 & 9.22120 \\
\hline \multicolumn{7}{|c|}{ (c) $F=110 \mathrm{kV} / \mathrm{cm}$} \\
\hline$N_{b}=1$ & -0.299997 & 0.371134 & 1.53208 & 3.30161 & 5.83361 & 9.15292 \\
\hline$N_{b}=4$ & -0.350466 & 0.325906 & 1.59092 & 3.33847 & & \\
\hline$N_{b}=5$ & -0.350496 & 0.323156 & 1.58650 & 3.30195 & 5.87733 & \\
\hline$N_{b}=6$ & -0.350513 & 0.323057 & 1.58547 & 3.30137 & 5.85058 & 9.18137 \\
\hline
\end{tabular}

setting $\mu=j$, and the $s$-radial symmetry, i.e., $m=0$, has been applied. The radial wave function to be determined satisfies the coupled equations

$$
-\frac{1}{2} A\left(\frac{d^{2}}{d \rho^{2}}+\frac{1 / 4}{\rho^{2}}\right) f^{E}(|\rho|)=(E I-\varepsilon) f^{E}(|\rho|)
$$

where matrix notations have been employed for $f^{E}=\left(f_{j \alpha}^{E}\right), \varepsilon$ $=\left(\varepsilon_{j} \delta_{j j^{\prime}}\right)$, and $I$ represents the unit matrix. Moreover, $A$ $=\left(A_{j j^{\prime}}\right)$ with

$$
A_{j j^{\prime}}=\left\langle\phi_{j}\left|1 / m_{\|}\right| \phi_{j^{\prime}}\right\rangle
$$

where $\phi_{j}$ is defined in Eq. (15), and it is understood that the integration is implemented over $z_{e}$ and $z_{h}$.

Equation (C4) has been numerically solved, resulting in the step-shaped spectra already shown in Fig. 9. In order to extract the underlying physics, it is preferable to solve Eq. (C4) in a closed analytic form. In view of the eigenvalue problem of $A$ : (a) $\mathrm{F}=90 \mathrm{kV} / \mathrm{cm}$

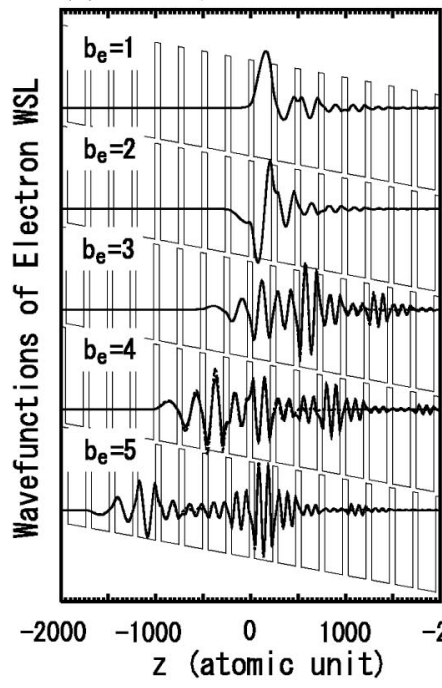

(b) $F=100 \mathrm{kV} / \mathrm{cm}$

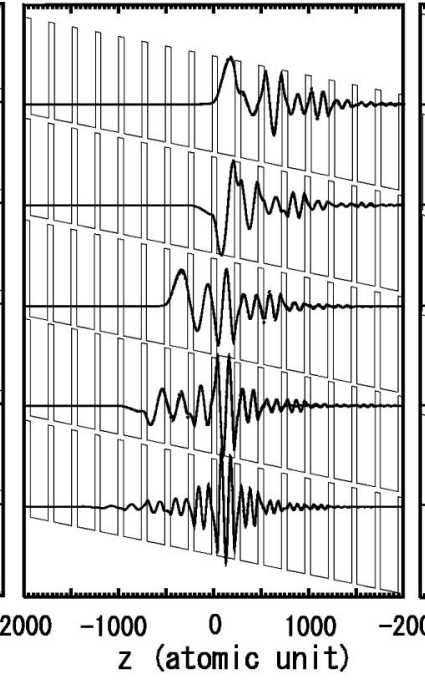

(c) $F=110 \mathrm{kV} / \mathrm{cm}$

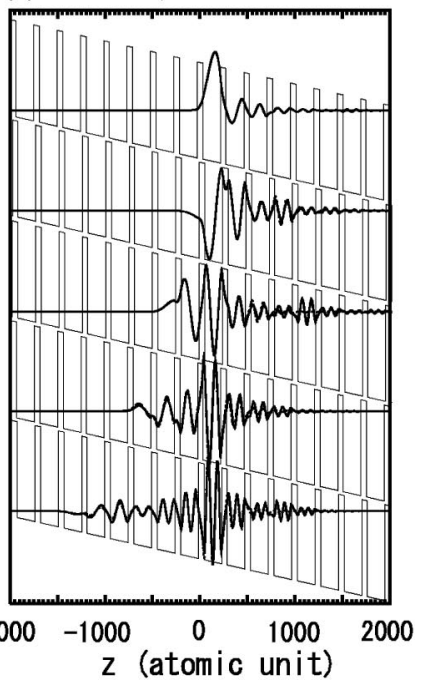

FIG. 14. The wave functions $\phi_{0\left(b_{e}\right)}^{(e)}$ of the coupled WSL of $e$ for $b_{e}=1-5$ at $F=(\mathrm{a}) 90 \mathrm{kV} / \mathrm{cm}$, (b) $100 \mathrm{kV} / \mathrm{cm}$, and (c) $110 \mathrm{kV} / \mathrm{cm}$. The traces indicated by the solid, dotted, and chain lines are obtained by using $N_{b}=6,5$, and 4 , with $N_{\text {site }}=20$, in order to confirm the convergence of the wave functions (WF- $N_{b}$ ) with respect to $N_{b}$. In the case of $b_{e}=5$, the traces with $N_{b}=4$ are missing because WF- 4 is not available. Only the solid lines can be seen from the present traces and the others are little discernible because the three sets of curve almost completely overlap. The geometry of the present WSL is specified in each panel. 


$$
A U=U \Lambda,
$$

where $\Lambda=\left(\lambda_{i} \delta_{i j}\right)$ is an eigenvalue matrix and $U$ is the unitary matrix associated with the set of eigenvectors, the resulting equation is

$$
-\frac{1}{2} \Lambda\left(\frac{d^{2}}{d \rho^{2}}+\frac{1 / 4}{\rho^{2}}\right) \tilde{f}^{E}(|\rho|)=(E I-\widetilde{\varepsilon}) \tilde{f}^{E}(|\rho|) .
$$

Here, $\widetilde{f}^{E}=U^{-1} f^{E}$ and $\widetilde{\varepsilon}=U^{-1} \varepsilon U$. $\widetilde{\varepsilon}$ can be expanded as $\widetilde{\varepsilon}=\varepsilon$ $-i[g, \varepsilon]+\cdots$, where $U=\exp (i g)$, and where $g$ is Hermitian. On the basis of the approximation that $\widetilde{\varepsilon}$ can be replaced by $\varepsilon$, Eq. (C7) can be decoupled. Solutions that ensure appropriate scattering boundary conditions are provided in terms of the Bessel function of the first kind, expressed as

$f_{j \alpha}^{E}(|\rho|) \approx|\rho|^{1 / 2} \sum_{j^{\prime}} U_{j j^{\prime}} J_{0}\left(k_{j^{\prime}}|\rho|\right) \delta_{j^{\prime} \alpha} C_{\alpha}=|\rho|^{1 / 2} U_{j \alpha} J_{0}\left(k_{\alpha}|\rho|\right) C_{\alpha}$,

where $k_{j^{\prime}}=\sqrt{2\left(E-\varepsilon_{j^{\prime}}\right) / \lambda_{j^{\prime}}}$, and $C_{\alpha}$ is a normalization constant denoted by $C_{\alpha}=1 / \sqrt{\lambda_{\alpha}}$ so that $f_{j \alpha}^{E}$ is energy-normalized. Therefore, the dipole moment $\mu_{\alpha}^{0}(E)$, corresponding to Eq. (9), is given by

$$
\mu_{\alpha}^{0}(E) \approx \sum_{j}^{N} w_{j}^{0} U_{j \alpha} C_{\alpha}
$$

where $J_{0}(0)=1$ has been taken into consideration and

$$
w_{j}^{0}=\int d z\left[\phi_{j}\left(z_{e}, z_{h}\right)\right]_{z \equiv z_{e}=z_{h}} .
$$

The photoabsorption spectra $I^{0}(E)$ are expressed as

$$
I^{0}(E)=\sum_{\alpha}^{N_{0}}\left|\tilde{\mu}_{\alpha}^{0}(E)\right|^{2}
$$

where

$$
\tilde{\mu}_{\alpha}^{0}(E)=\mu_{0} \mu_{\alpha}^{0}(E)
$$

*Electronic address: hino@bk.tsukuba.ac.jp

${ }^{1}$ G. H. Wannier, Phys. Rev. 117, 432 (1960).

${ }^{2}$ E. O. Kane, J. Phys. Chem. Solids 12, 181 (1959).

${ }^{3}$ W. V. Houston, Phys. Rev. 57, 184 (1940).

${ }^{4}$ J. Zak, Phys. Rev. Lett. 20, 1477 (1968).

${ }^{5}$ J. E. Avron, J. Zak, A. Grossman, and L. Gunther, J. Math. Phys. 18, 918 (1977).

${ }^{6}$ C. Zener, Proc. Phys. Soc., London, Sect. A 145, 523 (1934).

${ }^{7}$ H. M. James, Phys. Rev. 76, 1611 (1949).

${ }^{8}$ D. Emin and C. F. Hart, Phys. Rev. B 36, 7353 (1987); 41, 3859

(1990); 43, 2426 (1991); 43, 4521 (1991).

${ }^{9}$ L. Kleinman, Phys. Rev. B 41, 3857 (1990).

${ }^{10}$ D. A. Page and E. Brown, Phys. Rev. B 43, 2423 (1991).

${ }^{11}$ J. Zak, Phys. Rev. B 43, 4519 (1991).

${ }^{12}$ G. Nenciu, Rev. Mod. Phys. 63, 91 (1991).

${ }^{13}$ F. Bloch, Z. Phys. 52, 555 (1928).

${ }^{14}$ L. Esaki and R. Tsu, IBM J. Res. Dev. 14, 61 (1970).

${ }^{15}$ E. E. Mendez, F. Agullo-Rueda, and J. M. Hong, Phys. Rev. Lett. 60, 2426 (1988).

${ }^{16}$ P. Voisin, J. Bleuse, C. Bouche, S. Gaillard, C. Alibert, and A. Regreny, Phys. Rev. Lett. 61, 1639 (1988).

${ }^{17}$ V. G. Lyssenko, G. Valusis, F. Löser, T. Masche, K. Leo, M. M. Dignam, and K. Köhler, Phys. Rev. Lett. 79, 301 (1997).

${ }^{18}$ H. Schneider, H. T. Grahn, K. von Klizting, and K. Ploog, Phys. Rev. Lett. 65, 2720 (1990).

${ }^{19}$ M. Nakayama, I. Tanaka, H. Nishimura, K. Kawashima, and K. Fujiwara, Phys. Rev. B 44, 5935 (1991).

${ }^{20}$ A. Kristensen, P. E. Lindelof, C. B. Sorensen, and A. Wacker, Semicond. Sci. Technol. 13, 910 (1998).

${ }^{21}$ J. Leo and A. MacKinnon, J. Phys.: Condens. Matter 1, 1449 (1989).
${ }^{22}$ G. Bastard, R. Ferreira, S. Chelles, and P. Voisin, Phys. Rev. B 50, 4445 (1994).

${ }^{23}$ R. Ferreira, P. Voisin, and G. Bastard, Solid-State Electron. 37, 857 (1994)

${ }^{24}$ X.-G. Zhao, J. Phys.: Condens. Matter 4, L383 (1992).

${ }^{25}$ M. Helm, W. Hilber, G. Strasser, R. De Meester, F. M. Peeters, and A. Wacker, Phys. Rev. Lett. 82, 3120 (1999).

${ }^{26}$ S. Glutsch and F. Bechstedt, Phys. Rev. B 60, 16584 (1999).

${ }^{27}$ S. Glutsch, F. Bechstedt, B. Rosam, and K. Leo, Phys. Rev. B 63, 085307 (2001).

${ }^{28}$ S. Glutsch, Phys. Rev. B 69, 235317 (2004).

${ }^{29}$ H. Rosam, D. Meinhold, F. Löser, V. G. Lyssenko, S. Glutsch, F. Bechstedt, F. Rossi, K. Köhler, and K. Leo, Phys. Rev. Lett. 86, 1307 (2001).

${ }^{30}$ H. T. Grahn, H. Schneider, and K. von Klitzing, Phys. Rev. B 41, 2890 (1990).

${ }^{31}$ S. H. Kwok, H. T. Grahn, M. Ramsteiner, K. Ploog, F. Prengel, A. Wacker, E. Schöll, S. Murugkar, and R. Merlin, Phys. Rev. B 51, 9943 (1995).

${ }^{32}$ A. Sibille, J. F. Palmier, and F. Laruelle, Phys. Rev. Lett. 80, 4506 (1998).

${ }^{33}$ A. Sibille and J. F. Palmier, Appl. Phys. Lett. 60, 457 (1992).

${ }^{34}$ G. Scamarcio, F. Capasso, A. L. Hutchinson, D. L. Sivco, and A. Y. Cho, Phys. Rev. B 57, R6811 (1998).

${ }^{35}$ C. Rauch, G. Strasser, K. Unterrainer, W. Boxleitner, and E. Gornik, Phys. Rev. Lett. 81, 3495 (1998).

${ }^{36}$ A. D. Carlo, P. Vogl, and W. Pötz, Phys. Rev. B 50, 8358 (1994).

${ }^{37}$ M. M. Dignam and J. E. Sipe, Phys. Rev. Lett. 64, 1797 (1990); Phys. Rev. B 43, 4097 (1991).

${ }^{38}$ N. Linder, Phys. Rev. B 55, 13664 (1997).

${ }^{39}$ U. Fano, Phys. Rev. 124, 1866 (1961). 
${ }^{40}$ D. M. Whittaker, J. Phys. IV 5, 199 (1993); Europhys. Lett. 31, 55 (1995).

${ }^{41}$ C. P. Holfeld, F. Löser, M. Sudzius, K. Leo, D. M. Whittaker, and K. Köhler, Phys. Rev. Lett. 81, 874 (1998).

${ }^{42}$ T. W. Canzler, C. P. Holfeld, F. Löser, V. G. Lyssenko, K. Leo, D. M. Whittaker, and K. Köhler, Physica E (Amsterdam) 10, 593 (2001).

${ }^{43}$ J. M. Lachaine, M. Hawton, J. E. Sipe, and M. M. Dignam, Phys. Rev. B 62, R4829 (2000).

${ }^{44}$ R. B. Liu and B. F. Zhu, Phys. Rev. B 59, 5759 (1999).

${ }^{45}$ K. Yashima, K. Hino, and N. Toshima, Phys. Rev. B 68, 235325 (2003).

${ }^{46}$ K. Hino, K. Yashima, and N. Toshima, Phys. Rev. B 71, 115985 (2005).

${ }^{47}$ K. Hino, K. Goto, and N. Toshima, Phys. Rev. B 69, 035322 (2004).

${ }^{48}$ Discussion of the Zener tunneling effect on FR spectra of WSL excitons is made in Refs. 26-28.

${ }^{49}$ K. Hino and N. Toshima, Solid State Commun. 132, 449 (2004).

${ }^{50}$ K. Hino, Phys. Rev. B 62, R10626 (2000); 64, 075318 (2001).

${ }^{51}$ C. de Boor, A Practical Guide to Splines (Springer, New York, 1978), Chap. 9.

${ }^{52}$ A. S. Umar, J. Wu, M. R. Strayer, and C. Bottcher, J. Comput. Phys. 93, 426 (1991).

${ }^{53}$ D. F. Gallaher and L. Wilets, Phys. Rev. 169, 139 (1968).

${ }^{54}$ R. Shakeshaft, Phys. Rev. A 18, 1930 (1978).

${ }^{55}$ Q. Niu, X.-G. Zhao, G. A. Georgakis, and M. G. Raizen, Phys. Rev. Lett. 76, 4504 (1996).

${ }^{56}$ S. R. Wilkinson, C. F. Bharucha, K. W. Madison, Q. Niu, and M. G. Raizen, Phys. Rev. Lett. 76, 4512 (1996).

${ }^{57}$ M. BenDahan, E. Peike, J. Reichel, Y. Castin, and C. Salomon, Phys. Rev. Lett. 76, 4508 (1996).

${ }^{58}$ M. C. Fischer, K. W. Madison, Q. Niu, and M. G. Raizen, Phys. Rev. A 58, R2648 (1998).

${ }^{59}$ K. W. Madison, M. C. Fischer, R. B. Diener, Q. Niu, and M. G. Raizen, Phys. Rev. Lett. 81, 5093 (1998).

${ }^{60}$ In Figs. 2 and 4, it is remarked that there is no WSL state that has a zero dipole; i.e., no slope with respect to $F$ in regions where Zener tunneling is small. This is in contrast with previously published works. The difference comes from the $N_{\text {site }}$ used for calculations. The values of $N_{\text {site }}$ were selected to be even numbers in the present work $\left(N_{\text {site }}=20\right)$, while they were selected to be odd, for instance, in Ref. 37. However, the energy of a combined WSL of a pair of $e$ and $h$ is independent of the choice of $N_{\text {site }}$ since this is specified by the index of $n$ that is the difference between $n_{e}$ and $n_{h}$, i.e., $n=n_{h}-n_{e}$, as has been defined in Sec. I.

${ }^{61}$ L. I. Schiff, Quantum Mechanics, third ed. (McGraw-Hill, New York, 1968), p. 255.

${ }^{62}$ Strictly speaking, just a profile of FR composed of one-open and one-closed channels can be analyzed in terms of a single $q$ value that is commonly used for qualitative discussion.

${ }^{63}$ F. Mies, Phys. Rev. 175, 164 (1968).

${ }^{64}$ In the reference spectra of Fig. 9 using the Kane approximation, the redshift of the spectral peak ascribable to, for instance, the $0(1,1)$ subband state, characteristic of the quantum-confined Stark effect, is not observed. In the case of a system of the biased single quantum well, the redshift due to the quantumconfined Stark effect results from the Stark mixings of the lowest energy level of the original (field-free) quantum well with the higher excited energy levels. On the other hand, in the present Kane-approximated WSL case, there are no Stark mixings of the lowest miniband of the original (field-free) SLs with the higher minibands. This situation is equivalent to the approximation that the lowest energy levels of the original (field-free) quantum wells that the SLs are composed of are not coupled with the corresponding higher excited energy levels through the Stark mixing. This is why there is no redshift of the peak position in this case. For the quantum-confined Stark effect, see, for instance, D. A. B. Miller, D. S. Chemla, T. C. Damen, A. C. Gossard, W. Wiegmann, T. H. Wood, and C. A. Burrus, Phys. Rev. Lett. 53, 2173 (1984).

${ }^{65}$ K. Hino, J. Phys. Soc. Jpn. 67, 3159 (1998).

${ }^{66}$ The Coulomb coupling, for which the FR is responsible, also gives rise to interchannel coupling effects.

${ }^{67} \mathrm{~A}$ remark is made on whether valence-band nonparabolicity, arising from the valence-band coupling, alters, to some extent, the degree of magnitude of the reduced-mass mixing. By employing the Luttinger Hamiltonian [see, J. M. Luttinger, Phys. Rev. 102, 1030 (1956) and L. C. Andreani and A. Pasquarello, Phys. Rev. B 42, 8928 (1990)], instead of the kinetic energy part of Eq. (C2), we have performed the pilot calculations of the energy dispersion of the SLs of $h$ and the associated free spectra corresponding to both spectra $\mathrm{A}$ and $\mathrm{B}$ at approximately $F$ $=100 \mathrm{kV} / \mathrm{cm}$. These resulting free spectra in the region of $E$ $<\varepsilon_{0(1,1)}$, although not shown here, indicate tendencies toward the conspicuous redshift of the absorption tail edge in the region of the anticrossing; these tendencies are similar to those of the spectra represented by the filled and open squares in Fig. 10(c). This would lead to the conclusion that the valence-band mixing is so minor that the results of the reduced-mass mixing discussed in the text are only slightly modified. It is speculated that the valence-band nonparabolicity only contributes weakly to the reduced-mass mixing due to the fact that the energy region of $E<\varepsilon_{0(1,1)}$ concerned here is close to the edge of the joint $e-h$ band of the bulk GaAs $\left(E_{g}=1.519 \mathrm{eV}\right)$, where the parabolic band approximation is still correct. However, the following comment should be noted: In the single quantum-well system, the valence-band mixing gives rise to sizable shifts of FR peak positions of the order of $1 \mathrm{meV}$ [see, G. E. W. Bauer and T. Ando, Phys. Rev. B 38, 6015 (1988)] and intricate overlap resonance (see Ref. 50) when the exciton effect is taken into account; to the best of our knowledge, there is no calculation of the FR spectra of the WSL exciton based on the Luttinger Hamiltonian. In the case of the conduction-band nonparabolicity [see, M. F. H. Schuurmans and G. W. t'Hooft, Phys. Rev. B 31, 8041 (1985), and R. Eppenga, M. F. H. Schuurmans, and S. Colak, Phys. Rev. B 36, 1554 (1987)], this effect on the FR spectra would be considered negligible as far as the lower energy region close to $E_{g}$ is concerned, by consulting the oscillator strength of the exciton ground state discussed in the above reference of Bauer and Ando.

${ }^{68}$ See the paper included in Ref. 64.

${ }^{69}$ K. Hino, Solid State Commun. 128, 9 (2003).

${ }^{70}$ R. Winkler, Phys. Rev. B 51, 14395 (1995).

${ }^{71}$ K. Hino, J. Phys. Soc. Jpn. 71, 2280 (2002).

${ }^{72}$ D. Y. Oberli, G. Böhm, G. Weimann, and J. A. Brum, Phys. Rev. B 49, 5757 (1994). 